\title{
Be rich or don't be sick: estimating Vietnamese patients' risk of falling into destitution
}

\author{
Quan Hoang Vuong*
}

*Correspondence: qvuong@ulb.ac.be

Centre Emile Bernheim, Université Libre de Bruxelles, 50 Ave F.D. Roosevelt, Brussels 1050, Belgium

\section{黛 Springer}

\begin{abstract}
This paper represents the first research attempt to estimate the probabilities of Vietnamese patients falling into destitution due to financial burdens occurring during a curative hospital stay. The study models risk against such factors as level of insurance coverage, residency status of patient, and cost of treatment, among others. The results show that very high probabilities of destitution, approximately $70 \%$, apply to a large group of patients, who are non-residents, poor and ineligible for significant insurance coverage. There is also a probability of $58 \%$ that seriously ill low-income patients who face higher health care costs would quit their treatment. These facts put the Vietnamese government's ambitious plan of increasing both universal coverage (UC) to $100 \%$ of expenditure and the rate of UC beneficiaries to $100 \%$, to a serious test. The current study also raises issues of asymmetric information and alternative financing options for the poor, who are most exposed to risk of destitution following market-based health care reforms.
\end{abstract}

Keywords: Health insurance, Government policy on health care, Risk of destitution JEL Classification: 113, 118, 119

\section{Background}

Today, Vietnam has a population of 92 million, with a low per capita GDP of approximately $\$ 2000$. Financial hardship is common among the populace, both in urban and rural areas. The poverty issue is much more serious with families who have a seriously sick member.

On November 13, 2014, an article on Dan Tri-a popular online media source in Vietnam-reported on a story about patient Nguyen Thi Lan, in Thach Lap Commune, Giong Rieng District, Kien Giang (a southern province of Vietnam). She suffered from a serious brain tumour that led to uncontrollable behaviour and unintentionally dropped her 1-year-old daughter five times. Her family could not afford travel and health care costs, so they kept her home and used "traditional medicine" without success. That article made numerous readers empathetic to her family's plight; many sent money to help. On December 15, 2014, 47 million Vietnamese Dong (VND), approximately $\$ 2200$, was collected from various readers and sent to her family, allowing Mrs. Lan to travel to a provincial hospital and start treatment (Dan Tri Online 2014). Apart from showing

(c) 2015 Vuong. This article is distributed under the terms of the Creative Commons Attribution 4.0 International License (http:// creativecommons.org/licenses/by/4.0/), which permits unrestricted use, distribution, and reproduction in any medium, provided you give appropriate credit to the original author(s) and the source, provide a link to the Creative Commons license, and indicate if changes were made. 
public care about the hardship of their country fellows, this and similar articles also give rise to the issue of efficiency and use of health insurance, treatment costs and the general degree of financial destitution that many poor patients and their families face.

The amended Law on Health Insurance, effective January 2015, increases universal coverage (UC) to $100 \%$ of the population, providing a full coverage of all relevant expenditures. The state expects that the new law will help reduce exposure of members of society, especially the poor, to the risk of destitution-which has for decades been a harsh reality-caused by extreme medical care costs that uninsured patients have little choice but to pay.

Unfortunately, the problem is hardly new. More than 13 years ago, Whitehead et al. (2001) discussed the problem of patients risking falling into 'the medical poverty trap', giving a ballpark figure: "In rural North Vietnam, $60 \%$ of poor households were in debt, with a third citing payment for health care as the main reason". Also, the authors called for researchers and policy-makers to pay attention to poverty-alleviation strategies, bearing the medical costs to vulnerable sections of society in mind (p. 834-6). This research has attracted considerable attention from the public and scholarly communities, leading to more articles addressing this issue in developing countries. The need for further microeconomic research on the household costs of illness and implications for poverty is imperative: "International research efforts also need to develop a common illness cost and impact methodology to allow more meaningful comparisons of the economic burden of illness across settings and diseases" (Russell 2004: p. 152).

While highlighting important role of health economic evaluation (HEE) in strategic planning and policy making, Tran et al. (2014) reviewed 26 HEE studies in Vietnam and call for connecting researchers and policy-makers. Their findings of limitation of scope and number of works as well as severe technical errors or omissions imply a need for more empirical studies to promote evidence-based policies. There are also encouragements to supply policy-making process with stylised facts. Jelicic Kadic et al. (2014)call for using high-quality evidence in Croatian health care policy to rationalize expenditures and to ensure wider and better access to medicines. Zhang et al. (2015) consider China's National Reimbursement Ratio as a helpful quantitative indication in assessing and predicting national health insurance system. Santatiwongchai et al. (2015) affirm that there is room for improvement in the quality and usefulness of evidence to meet the need of governments and various development partners.

This article aims to identify factors that may affect the risk of destitution of Vietnamese inpatients based on a survey of patients who received inpatient hospital treatment. Many of the questions asked for perceptions of such critical determinants as severity of illness, distance of hospital from the patient's home, and "thank-you money" for allegedly premium health care and treatment.

The article begins with a literature review on studies of Vietnam's health care system, with an emphasis on insurance, costs and poverty. Next, it moves on to the research method of a baseline category logit model, which is employed to model the conditional probabilities of going destitute when certain specific events occur. The third section reports estimated results, together with computed probabilities, which address the research questions. The paper closes with a discussion of key insights and implications for patients, health service providers and the state. 


\section{Literature review}

Researchers have studied issues relating to health care systems, medical costs, the 'poverty trap', health reform policy-making and shed light on numerous aspects of lowincome countries' health care sector. This section briefly discusses issues related to the Vietnamese health care sector, which give rise to the research questions.

\section{Health care reforms and financing issues}

Bloom (1997) sees the need for 'radical health sector reforms' for low-income countries, and states that China and Vietnam could exemplify a model of financing health services, especially in rural areas. However, both countries face the issue of rising health costs and inequalities among groups of different income levels. In the 1990s, a high proportion of rural people in Vietnam were able to consult with health workers in the community, and to Bloom: "This suggests that access to basic health services is reasonably good". Still, understanding the impact of illness on risk of becoming financially distressed is more challenging due to scarcity of socioeconomic data. Quality of information for policy making is thus limited and seriously affected. In addition, development of financing mechanisms that assist in covering treatment costs has seen little progress and is still an issue for debate. Medical costs usually serve to be a 'shock' to household's well-being.

Also in China, after a decade of reforms to significantly broaden government-backed insurance coverage and the availability of basic care, Daemmrich (2013, p. 1) notices the Chinese Government "are encountering a dilemma between supporting profit-seeking industries that offer the potential for new medical products and services but want freemarket pricing, and public access to low-cost care that requires redistributive policies and price controls to function efficiently". If one considers Vietnam's reforms of health care system has started with amendment of the Law on Health Insurance and recent growth of private hospitals (Hort 2011) then overcoming such a dilemma is a challenge to the country's policy makers. To this end, quantitative indications of financial matters-including probability of falling in destitution and factors that determine the probability - are helpful for both public and private players to do cost-benefit analysis.

Coping with rising medical costs, in either normal illness or a catastrophic event, means dealing with issues of increasing levels of debt and without understanding the probability of falling into a poverty trap, it will be hard to devise effective strategies for households to mitigate the risk of falling into financial hardship (Russell 2004: p. 153) as things have changed as the market modus operandi comes into play. Bloom (1997: p.16) provides some useful statistics: the richest quartile of rural Chinese spend 3.2 times as much on medical care as the poorest quartile; the figure for Vietnam was 4.6 times in 1994. Health care charges have become a burden for the poor, with rural Chinese spending up to five times the average daily per capita income on an average prescription. Vietnamese are spending $8 \%$ of their annual non-food consumption for each visit to a commune health care station (Bloom 1997: p.16). The risk of falling into financial hardship jumps when there is a seriously ill family member, as average hospital admission could cost $60 \%$ of the annual net income of poor households in China. Moreover, an average commune health unit admission costs $45 \%$ of a poor family's annual non-food consumption in Vietnam. An adverse health event can cause increasing debts and asset sales, and becomes an important cause of poverty. The poor have too 
few financing options. What is more, economic reforms have led to a situation in which the relationships between health workers, government and patients is altered, and health service providers now favour the rich, to whom they can supply expensive drugs and sophisticated technologies (Bloom 1997: pp. 17-18).

Regarding financing alternatives for the majority of patients, Sepehri et al. (2003) verify that Vietnam's health care system has undergone major structural reforms, which significantly affect the delivery and financing of health services. Emerging issues are access, efficiency and equity in health services sector, and the trend of dwindling state funds and a shift from state financing to out-of-pocket fees paid by patients (p. 156). The rich tend to receive more health care, with longer hospital stays, and use more intensive resources than do the poor. The poor receive proportionally less care, with a rising trend of overprovision of services and expensive drugs, leading medical care costs to take up a larger percentage of a family overall income.

Specifically, Lönnroth et al. (2001) point to the fact that 'evening clinic'-a kind of privately run health service operation used by out-patients-treatment of tuberculosis by private physicians may cost 200,000-1,000,000 Vietnamese Dong/month (\$13-\$67). For many households, that amount is a 'heavy' financial burden. Apart from fees and drugs, patients and household members were also worried about travel costs and time-consuming processes that usually triggered discontinued income during treatment periods, which could exceed fees and the costs of drugs (Lönnroth et al. 2001: p. 940-3).

In a broad and highly influential study, Whitehead et al. (2001) unveil that poor households reporting illness in a rural area in northern Vietnam spent on average $22 \%$ of their household budget on health-care costs, whereas rich households spent $8 \%$ (p. 834). In this report, the authors do not state explicitly the definition of 'rich' and 'poor' patients and rather refer to the World Bank's classification. That is why 'home remedies' are still a preferred choice among the poor, representing 'the cheapest healthcare option' although the average cost rose progressively due to the price of drugs and consultations (Segall et al. 2002: p. 500). While Segall et al. (2002) note that non-poor households spent on average $150 \%$ of their monthly income, the lowest cost by the poor represented $200 \%$ of their monthly income. Nonetheless, due to the income gap between the two groups, on average, non-poor households spent much more than the poor per admission in value. In rural areas of Vietnam, 3.3-10\% of the annual income per capita was devoted to health care-while an average of $2-7 \%$ was typical in a variety of developing countries-leading many Vietnamese households to also sell rice reserves and livestock, apart from borrowings, to finance health costs (Segall et al. 2002: pp. 501-2). Therefore debt, as a major financing option for healthcare services, remained pervasive among the poor.

In the same vein, Ha et al. (2002) confirm the burden on households in rural areas and report that severely ill people tend to use public care (p. 61), although public services showed a tendency to consume more resources than private services, that in part means these services tend to cost more. The authors estimate that the amount of subsidy was quite small, in fact negligible, accounting for around $4 \%$ the of total expenditures (pp. 67-8). Also, new issues emerge to exacerbate the problem of the financial burdens of health care, as Ensor (2004: p. 245) adds, "there is growing evidence to suggest that unofficial health care fees are likely to distort health care priorities and change the impact of health system reform" in developing countries. This also applies to the 
situation of Vietnamese health care sector as confirmed by results reported by Nguyen et al. (2012) upon surveying 706 households in 2008.

As to factors giving rise to the risk of poverty, Sepehri et al. (2005) postulate a possible link between income and length of hospital stay, as in transition economies post-hospital follow-up is virtually non-existent and travel is costly. According to the authors, a longer stay may increase assurance, reduce post-treatment complications and readmission, or simply speaking: better-quality care (p. 97). They suggest further investigations to examine the effects of unofficial and official payments on the intensity and quality of health care (p. 98) and the differences between groups of patients. This postulation by Sepehri et al. (2005) appears to be relevant to observations of Vietnamese patients and worth looking at. On the one hand, due to inadequate facilities some upper-tier hospitals such as Viet Duc have the policy of providing intensive care for most cases so that the length of stay for in-patients reduces to 7 days, whenever possible. One the other hand, there is certainly evidence of unnecessary in-patient care and excessive length of stay encouraged by other hospitals, aimed at higher average revenue collected per patient. There is no significant difference between health care fees between the poor and non-poor in public health services; it is likely that public sources may subsidise the rich rather than the poor (Thuan et al. 2008: p. 7). In light of this, Ekman et al. (2008: p. 252) conclude that there is an imperative need for reforming Vietnamese health insurance to focus on: (1) sustained resource mobilisation; (2) comprehensive functions of the health financing system; and (3) a long-term view of health insurance reform. Although roughly $50 \%$ of the population benefit from some form of health insurance, only $18 \%$ of the poor are entitled to these limited benefits, mainly channelled through the so-called Health Care Funds for the Poor (HCFP); 3/4 of which come from the central government and 1/4 come from a provincial source (Ekman et al. 2008: p. 255). The reality is that voluntary health insurance is still not easy and exhibits the asymmetric information issue.

What we learn from the extant literature is that although market reforms improve availability of health services, financing issues have arisen due to the tendency of inflating health care costs, in many cases unnecessarily. Debt financing for seeking health services has been common, especially among the poor, which subsequently increases the possibility of going destitute.

In addition, while emphasizing financial burden of medical care on the poor (Sepehri et al. 2003, 2005; Segall et al. 2002), especially patients who come from rural areas (Bloom 1997; Whitehead et al. 2001; Ha et al. 2002; Nguyen et al. 2012), the authors suggest distance from patient's home to treatment facilities matters. Lönnroth et al. (2001: p. 940), indeed, take a note on the cost of travel.

In developing economies, trying to access to urban health care services is a common practice of rural patients. Bronstein and Morrisey's work (1991) on data from 1983 and 1988 on hospital use in Alabama (USA) provides empirical evidence for increasing proportion of rural pregnant women travelling to metropolitan areas for infant services. Parkhurst and Ssengooba (2009) tell the same story in Uganda. Buczko (1994) affirms that rural hospitals are often bypassed by aged patients. The reasons may include avoiding assumingly inadequate care and accessing to advanced medical procedures. Moreover, Paul (1999) reports on widespread incidence of national health care bypassing in Bangladesh. Bangladeshi patients prefer foreign health care services because of lower 
costs, availability of specialized care, and better quality of services. Leonard et al. (2002) also consider strong preference of quality as a major reason for bypassing in Tanzania.

In Vietnam, the enforcement of amended Law on Health Insurance commencing on 1 January 2015 makes bypassing a burning issue. Although the Vietnam Ministry of Health unveils that 70 per cent of bypassed treatments are unnecessary (Nam Phuong 2015) and bypassed patients are eligible for much lower insurance payment [in comparison to previous regulation] the amendment is reportedly fail to prevent bypassing. Hospitals in economic hub Ho Chi Minh city reported a surge of patients declaring "non-insurance". Oncology Hospital in the city noticed the number of non-insurance patients went up by 250 per cent. Many insured patients decide to declare uninsured since the insurance payment is so little in comparison to other expenses such as travelling and accommodation for family members who escort the patients during treatment period], a representative of the Hospital told Tien Phong Newspaper (Quoc Ngoc 2015).

\section{Use of health services, costs and insurance benefits, and treatment outcome}

As health sector reform takes place, user fees grow. A major problem with user fees is that, although they help relieve the financial burden on the government, these fees can drive people into poverty and widen the gap between the rich and the poor. The need to establish measures for protecting the poor is imperative, especially in eliminating unofficial payments and asymmetric information between providers and patients. While only a small proportion of rural residents are eligible to receive health insurance benefits, low insurance coverage also increases the burden on the poor (Dao et al. 2008: pp. 1076-7).

Another issue is that statistics may have been biased due to the finding that the poor are likely to "modify the perception of sickness" to avoid costs due to health care needs and discontinued income (Thuan et al. 2008: p. 5). The poor show a higher tendency of using self-treatment, while the expenditure for self-treatment is only $13 \%$ of the total curative expenditure. A possible explanation of this low expenditure ratio is because actual self-treatment costs tend to be under-reported.

Regarding health insurance, Liu et al. (2012) report significant differences in health insurance coverage between Vietnam and China (employing a data set containing observations from two provinces at different levels of economic development, Shandong and Ningxia) although the two countries share similar systems and socio-economic properties. Through a survey of six counties in China, the authors reported coverage rates ranging from 85 to $91 \%$, but the rate is much lower in Vietnam, which is about $50 \%$, including both voluntary and compulsory schemes. Still, while insurance coverage levels may be high in rural China, the benefit package is limited and co-payment ratio is high, disadvantaging the poor. Dang et al. (2006) offered a detailed comparison between the Chinese and Vietnamese. Vietnamese patients with health insurance are significantly more likely than uninsured to utilise in-patient services (Liu et al. 2012: p. 5). Vietnamese perceive that the insured receive poorer quality of services than non-members, reflecting their complaints that using insurance leads to prescription of only limited types and amounts of medicine and longer waiting time. Thus, it is quite common that insured patients go to private drug sellers for medicines that are ineligible under the public scheme (Liu et al. 2012: p. 6). With respect to the common practice of using private healthcare providers, a ready explanation is because patients are not seriously ill 
and therefore do not require complicated process of treatment. However, there are other factors also taken into account in making such decisions: (a) inadequate understanding of the risk of inappropriate treatment; (b) convenience for patients' relatives; and, (c) trust on 'rumors' about reputation and efficacy of treatment methods by some local physicians, especially in rural areas where the use of traditional medicines (including herbal medicines) is common.

The relationship among the variables of use of health services, costs and insurance coverage is anticipated. Nonetheless, the impact of these factors, specifically costs and insurance coverage, on the treatment outcome is not obvious partly because they depend on the criticality of the patients when hospitalized. Thus, it is difficult to generalize the relationship, and there is little discussion on this specific issue.

\section{'Sensitive' issues relating to out of pocket (OOP) payment}

Regarding financing mechanisms in developing countries, the 'implied' risk of inflating the financial burden has become clearer with unreported out-of-pocket (OOP) payments by patients. Van Doorslaer et al. (2006) surveyed eleven low income countries and found that in Vietnam (as well as Bangladesh, China, India, and Nepal), more than $60 \%$ of health care costs are paid out-of-pocket, and OOP health payments exacerbate poverty (p. 1357). Moreover, $2-7 \%$ of the population in the eleven countries may fall below the extreme poverty threshold (\$1/day) due to health care payments. The authors also suggest country policy makers conduct evaluations to learn more about specific reforms in health financing that could help reduce impoverishment due to health care payments (pp. 1362-1364).

Again, Van Doorslaer et al. (2007) found that the OOP share remains highest in Bangladesh, India and Vietnam, with 10.6-12.6 \% of non-food expenditures spent on health care (p. 1169). These same three economies also continue to have the highest incidence of catastrophic payments (p. 1173). Chaudhuri and Roy's (2008: pp. 42-44) report that OOP payment is positively related to per capita consumption, and increases for higher consumption quintile, revealing differences in the redistributive effect, the additional costs due to OOP payment would likely deter the Vietnamese poor from seeking health services.

In countries with such high levels of catastrophic healthcare expenditure and significant OOP payment, Xu et al. (2007) suggest a need to move away from OOP payments, using prepayment systems, 'financial risk protection strategies', and increasing funds for alleviating social inequalities in health care (pp. 981-982). In India, Karan et al. (2014) report that financial burden of OOP spending increases faster among disadvantaged groups, in comparison to the more advantaged or wealthy.

In Vietnam, the OOP issue has become even more 'sensitive' as more retired state employees are affected. They had used the state-subsidized healthcare system and been covered almost fully. For the rest of the society, the OOP payment requires paying bribes to doctors, nurses and hospital staffs in hopes for better care. In fact, Vietnamese patients tend to regard the OOP to cover extra medicine as the 'new normal' but remain highly uncomfortable with OOP 'envelops', although this practice has become widespread. The issue has been regarded as 'sensitive' (everybody knows but nobody tells) in transition economies like Vietnam and China, where health care infrastructures are 
inadequate and underinvested, and generally inefficient. The issue of "thank you money" as part of the expected OOP payments can become highly political, too.

The literature review suggests that researchers agree on: (1) the need for alternative financing for patients in developing countries, in particular Vietnam, and especially for the poor; (2) the implied risk of falling into destitution is high, especially for the poor; (3) there is a pressing need to better understand the relationships between socio-economic factors that help explain financial distress faced by the poor; and, (4) there is inadequate protection, at least via the health insurance system, for the poor. This suggests the need for empirical investigations to examine financing issues, illness, insurance, end result of treatment, health care costs, length of stay, 'envelope OOP' and the probability of post-treatment destitution, for different groups of patients. Although not all factors will have simultaneous or equal effects on the post-treatment financial conditions and treatment result, the research suggests likely relationships among several. That is what this study sought to explore.

\section{Research questions and method}

Although the existing research significantly contributed to the understanding of the Vietnamese health care systems and issues with patients' hardship, there is little about the probability of patients falling into destitution. In addition, little research examines the factors that enhance risk to patients when they have to decide whether to use health care services. Such insights could inform the policy making process in Vietnam by identifying critical factors and directions for improvements.

\section{Research questions}

Improving the understanding of the Vietnamese health sector and patients' risks involves answering the following research questions (RQ), which would complement existing knowledge and may contribute to upcoming health sector reform:

RQ1: Does residency status of patients and insurance coverage determine the probability of patients falling into indebtedness? The specific factor of residency status is important in Vietnam because society has for long been skeptical about provincial healthcare, leading patients to travel to major urban hospitals in Hanoi, Hai Phong, or HCMC. Doing so involves the travel costs, care taking that family members must provide and informational asymmetry about drug prices, treatment schedules, the best hospital to visit and even 'right amount' of "extra thank-you money" OOP.

RQ2: As for two most important factors to Vietnamese patients/households, i.e. treatment costs and illness, is there evidence to support this view and if yes, whose influence better explains the possibility of end results of treatment, empirically?

RQ3: Can the likelihood of paying too little or too much out-of-pocket "extra thankyou money" be determined by the severity of illness and/or income of patients? This OOP amount may be significant but if a patient appreciates the value of service, he/she would be willing to pay depending on his/her availability of finance, before or after the course of treatment.

\section{Research method}

The multi-category logit models (also known as, polytomous logistic regression analysis) will be used to investigate the RQ1-3; the resulting models show behaviours 
of multinomial response variable $(Y)$ following multinomial (and binomial) predictor variables.

The specific analysis employed in this article is baseline-category logits (BCL). This type of modelling enables us to detect relationships between discrete variables, and in this kind of survey, likely polytomous response variables and discrete (multinomial or binomial) explanatory variables. In addition, it allows us to compute useful probabilities upon specific events of hypothetical influence.

Although log-linear models are also useful in modelling this type of problem, logistic regression is preferred due to: (1) fewer and thus more significant variables and (2) direct interpretation of the estimated coefficients in measuring the empirical probabilities of events. Moreover, BCL models provide a simultaneous representation of the odds of being in one category relative to being in a designated category, called the baseline category, for all pairs of categories.

In this investigation, a patient (among $n$ patients) can be regarded as independent and identical, and may have outcome in any of $J$ categories for each factor to be investigated. Let $y_{i j}=1$ if patient $i$ has outcome in category $j$ and $y_{i j}=0$ otherwise. Then, $\mathbf{y}_{i j}=\left(y_{i 1}, y_{i 2}, \ldots, y_{i c}\right)$ represents a multinomial trial, with $\sum_{j} y_{i j}=1$. Denote $n_{j}=\sum_{j} y_{i j}$ the number of "trials" having outcome in category $j$, the count $\left(n_{1}, n_{2}, \ldots, n_{c}\right)$ have a multinomial distribution. Let $\pi_{j}=P\left(Y_{i j}=1\right)$ denote the probability of outcome in category $j$ or each patient, then the multinomial probability mass function is computed as follows:

$$
p\left(n_{1}, n_{2}, \ldots, n_{c}\right)=\left(\frac{n !}{n_{1} ! n_{2} ! \cdots n_{c} !}\right) \pi_{1}^{n_{1}} \pi_{2}^{n_{2}} \cdots \pi_{c}^{n_{c}} .
$$

This distribution has the following properties:

$$
\begin{aligned}
& \mathrm{E}\left(n_{j}\right)=n \pi_{j} \\
& \operatorname{var}\left(n_{j}\right)=n \pi_{j}\left(1-\pi_{j}\right) \\
& \operatorname{cov}\left(n_{j}, n_{k}\right)=-n \pi_{j} \pi_{k} .
\end{aligned}
$$

where $\sum_{j} n_{j}=n$.

Now, let $\pi_{j}(\mathbf{x})=P(Y=j \mid \mathbf{x})$ represent a fixed setting for predictor variables, with $\sum_{j} \pi_{j}(\mathbf{x})=1$. Count data are grouped into $J$ categories of $Y$ as multinomial with corresponding sets of probabilities $\left\{\pi_{1}(\mathbf{x}), \ldots, \pi_{j}(\mathbf{x})\right\}$.

The baseline category logit models align each response (dependent) variable with a baseline category, taking the form:

$$
\ln \frac{\pi_{j}(\mathbf{x})}{\pi_{j}(\mathbf{x})}=\alpha_{j}+\boldsymbol{\beta}_{j}^{\prime} \mathbf{x}, \quad j=1, \ldots, J-1 .
$$

BCL analysis simultaneously models the effects of $\mathbf{x}$ on $(J-1)$ logits, which in general vary according to the response paired with the baseline category. The estimating of $(J-1)$ equations employing a given empirical data set would provide for parameters for these logits, as:

$$
\ln \frac{\pi_{a}(\mathbf{x})}{\pi_{b}(\mathbf{x})}=\ln \frac{\pi_{a}(\mathbf{x})}{\pi_{J}(\mathbf{x})}-\ln \frac{\pi_{b}(\mathbf{x})}{\pi_{J}(\mathbf{x})} .
$$


The empirical data set, which contains count data and mainly uses categorical variables, would enable the computing of Pearson-type likelihood ratio test statistics $\left(X^{2}, G^{2}\right)$ or goodness-of-fit.

The polytomous logistic model is estimated as a multivariate generalized linear model (GLM) which takes the form:

$$
\mathbf{g}\left(\boldsymbol{\mu}_{i}\right)=\mathbf{X}_{i} \boldsymbol{\beta},
$$

where, $\boldsymbol{\mu}_{i}=\mathrm{E}\left(\mathbf{Y}_{i}\right)$, corresponding to $\mathbf{y}_{i}=\left(y_{i 1}, y_{i 2}, \ldots\right)^{\prime}$; row $h$ of the model matrix $\mathbf{X}_{i}$ for observation $i$ contains values of independent variables for $y_{i h}$. For a BCL model, $\mathbf{y}_{i}=\left(y_{i 1}, y_{i 2}, \ldots, y_{i, J-1}\right)^{\prime}$; thus $y_{i J}$ is redundant. Therefore, for BCL: $\mu_{i}=\left(\pi_{1}\left(\mathbf{x}_{i}\right), \pi_{2}\left(\mathbf{x}_{i}\right), \ldots, \pi_{J-1}\left(\mathbf{x}_{i}\right)\right)^{\prime}$.and,

$$
g_{j}\left(\boldsymbol{\mu}_{i}\right)=\ln \left\{\mu_{i j} /\left[1-\left(\mu_{1}+\cdots+\mu_{i, J-1}\right)\right]\right\} .
$$

A rich account of technical details for practical modeling of polytomous logistic models is provided in Agresti (2002: pp. 267-74). Actual estimations performed in this study-whose results are reported in the next sections-employ analysis in R, following a set of instructions provided by Penn State at https://onlinecourses.science.psu.edu/stat504/node/171.

As a main purpose of the estimation is to compute response probabilities from multinomial logits, i.e. $\left\{\pi_{j}(\mathbf{x})\right\}$, the following computation will apply:

$$
\pi_{j}(\mathbf{x})=\frac{\exp \left(\alpha_{j}+\boldsymbol{\beta}_{j}^{\prime} \mathbf{x}\right)}{1+\sum_{h=1}^{J-1} \exp \left(\alpha_{h}+\boldsymbol{\beta}_{h}^{\prime} \mathbf{x}\right)} .
$$

with $\sum_{j} \pi_{j}(\mathbf{x})=1 ; \alpha_{J}=0$ and $\boldsymbol{\beta}_{J}=0$. The computed probabilities can be used to model the risk of a patient to fall into a category of financial distress (indebtedness or destitution) conditional upon some other "events" such as "being in the lower socio-economic status group" (SES) and/or "being non-resident" as to where the hospital is located, and/or "being insured", and so on.

\section{The data set and estimations}

\section{The survey, data and description}

The survey was conducted by a team including hospital personnel and a Hanoi-based research firm, collecting data from inpatients of many hospitals in northern Vietnam including but not limited to: Viet Duc Hospital, Bach Mai Hospital, Vietnam-Japan Hospital, Hai Duong Polyclinic Hospital, Thai Binh Polyclinic Hospital, Ministry of Transports Polyclinic, to name just a few.

Interviewers approached patients individually and gradually acquired information for the survey, including questions about "sensitive data" that a more general/social survey could hardly obtain. Such questions included family status, patient's income level, patient's extra expenses to doctors and hospital's staff, and their borrowings money to finance treatment (Additional file 1).

The research team obtained qualified data for 330 patients, from a total of approximately 1000. The data team consists of six people, one in charge of coordinating and checking quality, two in putting data into the database, and three of data collecting from hospital sources. These 1000 interviewees were selected randomly from the 
hospital records and based on the judgement by data collecting people about whether the patient/relative is available and/or willing to participate, after explaining about the ethical standards, issues of information nondisclosure and the possible insights the survey may contribute to the understanding of policy-makers and public in general. Sometimes a respondent had been approached multiple times over few weeks before he/she agreed to answer the survey completely. Nearly 400 participated and only 330 were considered of satisfactory quality for the subsequent analysis. The survey process started in first week of August 10, 2014 and ended first week of February 2015 (Additional file 2).

The following variables directly or indirectly enter into the analysis process:

- "Res": if a patient is considered to be a "resident" of the region where a hospital is located;

- "Stay" if a patient's stay in the hospital is less than 10 days ('short') or equal-orgreater-than 10 days ('long')

- "Insured" if a patient is entitled to some insurance coverage under the UC or specific coverage provided by an employer;

- "SES" had four levels of socioeconomic status for the patient/household: very high/ rich; high; medium; low;

- "Illness" representing the severity of sickness of the patient when hospitalised

- "IncRank" showing the income level of a patient;

- "Spent" and "Dcost" represent amounts spent during the treatment period and average daily cost paid by the patient, in millions of Vietnamese Dong (VND 1 million $=\$ 47.2)$;

- "Pins; Pinc; Pchar; Ploan”: percentage of payment by sources of insurance coverage, savings, charity funds, and borrowings, respectively;

- "Streat, Srel, Senv": percentage of spending on direct treatment costs, relatives and friends for caring for the patient, "thank-you envelops", and OOP, respectively;

- "Burden": levels of financial burden on the patient/household following treatment; and,

- "End": end outcome of treatment telling if the patient fully recovered, partially recovered, stopped treatment in the middle of the process, or stopped treatment earlier due to lack of financing options.

Detailed information for all variables and their categorical values are provided in "Appendix 1".

An empirical distribution of income and hospital stay among patients constituting the sample is shown in Fig. 1. In the dataset, these three factors are represented by the quantitative variables Age, Days and Income [in millions of Vietnamese Dong (VND) per year]. A large portion of the sample is constituted by inpatients that stayed less than 10 days in the hospital. In addition, a large portion of patients have incomes lower than VND 50 million (approximately \$2360) per year, and patients with annual incomes below $\$ 4720$ account for more than $90 \%$ of the sample (see Fig. 1a). Likewise, the majority of patients stay less than 10 days in the hospital (Fig. 1b).

Figure 2 presents sources of financing for paying health care costs by patients, from insurance policy reimbursement (Pins) to savings from the patients and their family members (Pinc). These are also quantitative variables measured in percentages. Clearly, 


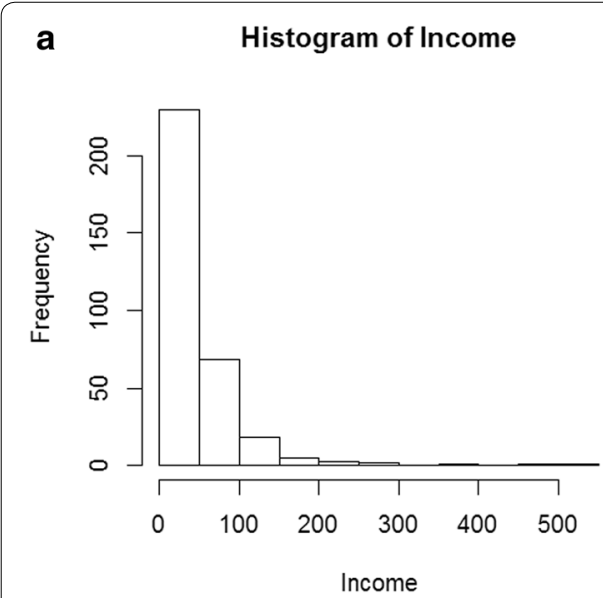

b

Histogram of Days

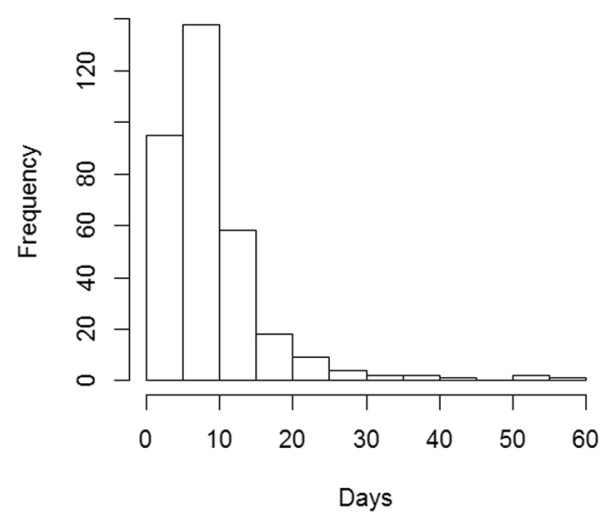

Fig. 1 Frequency distribution of patients'age and time spent in the hospital. a-b Two histograms for patients survey with respect to income levels and corresponding stays in hospital

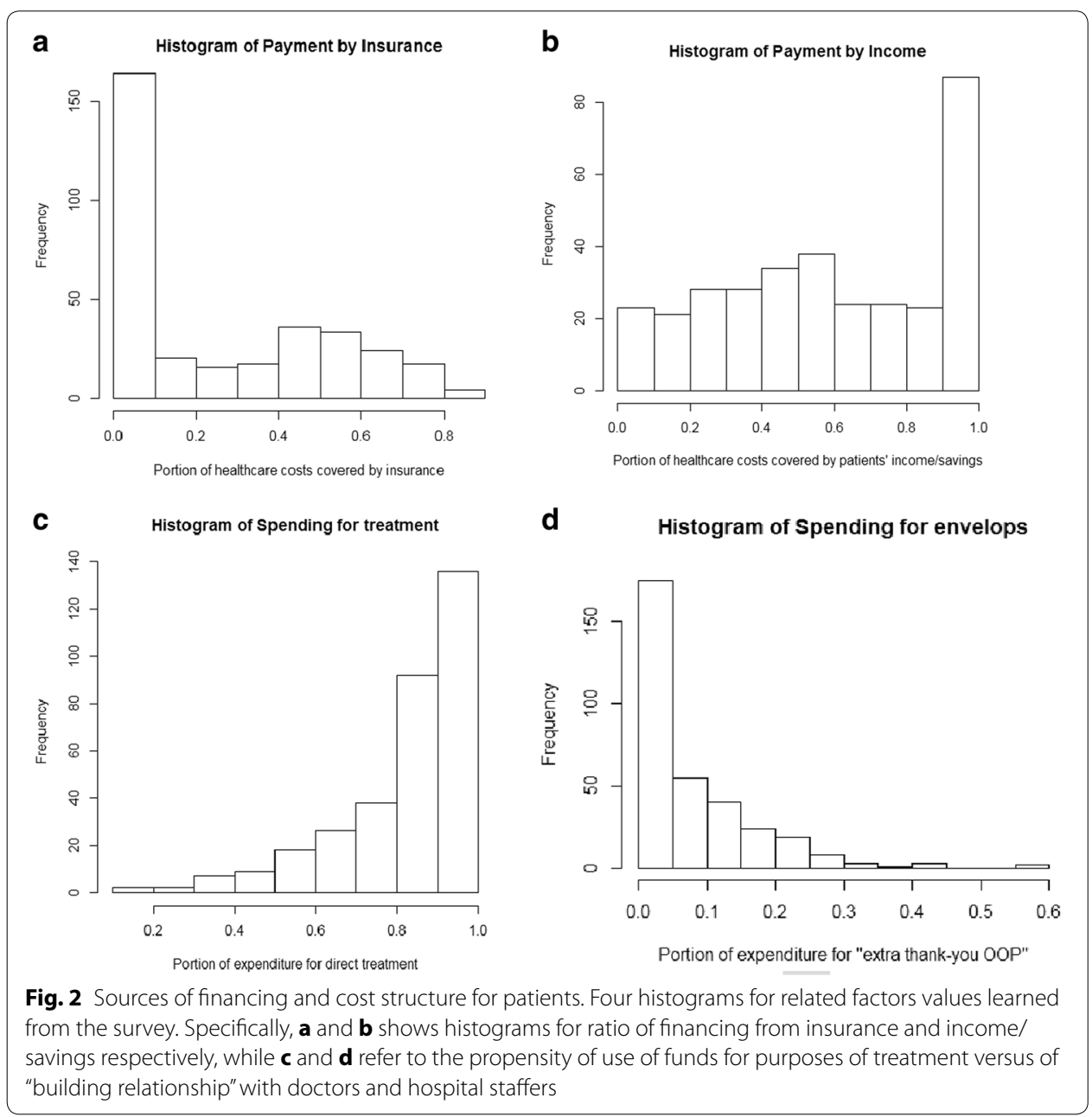

a majority of surveyed patients receive less than $50 \%$ reimbursement from their insurance coverage; income/savings represents the single most important financing source for paying health care costs for the majority of patients (Fig. 2a, b). 
Likewise, the "Histogram of spending for treatment" in Fig. 2 shows the frequency of patients paying for the main costs of treatment (e.g., hospital room, medicines, use of equipment, nurse care); "Spending for envelops" is the portion of a patient's total payments for extra costs to doctors and hospital's staff in the popular form of "envelop" (thank-you money and/or bribe). It can be seen that the majority (80-100\%), of patients' expenses are for direct treatment costs and hospital services, while the majority of patients pay less than $15 \%$ of the total expenses for "thank-you envelops", thus "portion of expenditure" for "extra thank-you OOP" $>15 \%$ is considered to be a high portion of an OOP payment.

Figure 3 represents data points, each with 3 numerical values of average daily cost (horizontal axis; in millions of Vietnamese Dong per day; VND 1 million $\sim \$ 47.2$ ), total health care expenses for the treatment (vertical axis; in millions of Vietnamese Dong) and number of days in the hospital (taking the natural logarithm to reduce the difference in effect size for better visualisation). The differences among patients are quite substantial.

In Fig. 4, those who were most likely to require longer hospital stays naturally divided into two groups, residents and non-residents. Generally speaking, people coming from other provinces tended to stay a little longer than those from within the region. However, the difference is not very large and likely insignificant. For each group, the dispersion of length of stay was large. In the subsequent analysis, more than a 10-day stay is considered "longer".

Next, Fig. 5 provides two graphs for the distribution of total expenses and average daily costs per patient, divided into groups of patients with different end results of treatments (A: full recovery; B: partial recovery; C: stopped in middle; D: unsuccessful treatment, including mortality). Both total expenses and average daily costs are on the vertical axis, and measured in millions of Vietnamese Dong (VND 1 million $=$ US \$47.2 using the official exchange rate as of Oct 15, 2014).

For both factors of expenditure and daily cost, the most varying range belongs to group D. There exist outliers if actual monetary values of expenses and daily cost are used. Thus, the choice of categorical data becomes more appropriate.

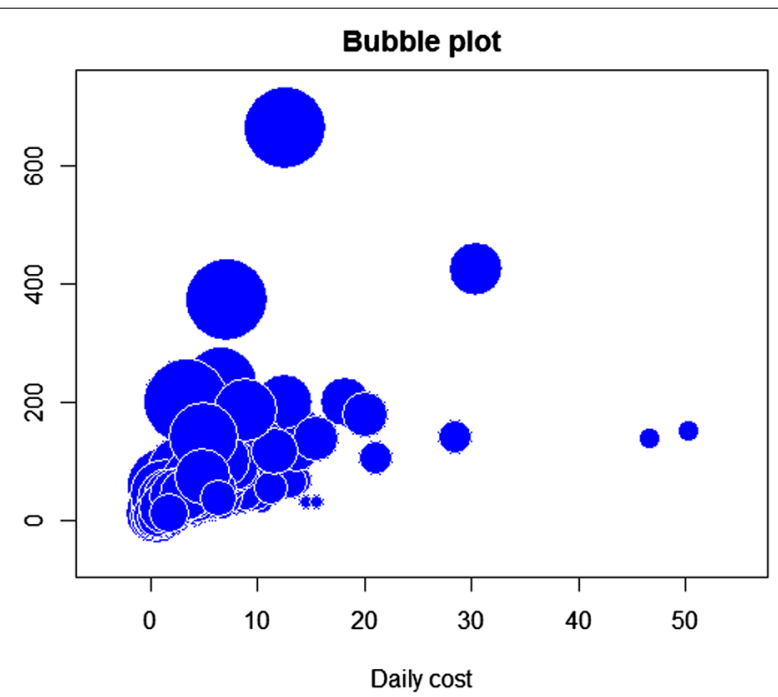

Fig. 3 Daily cost, total expenses and days in hospital 

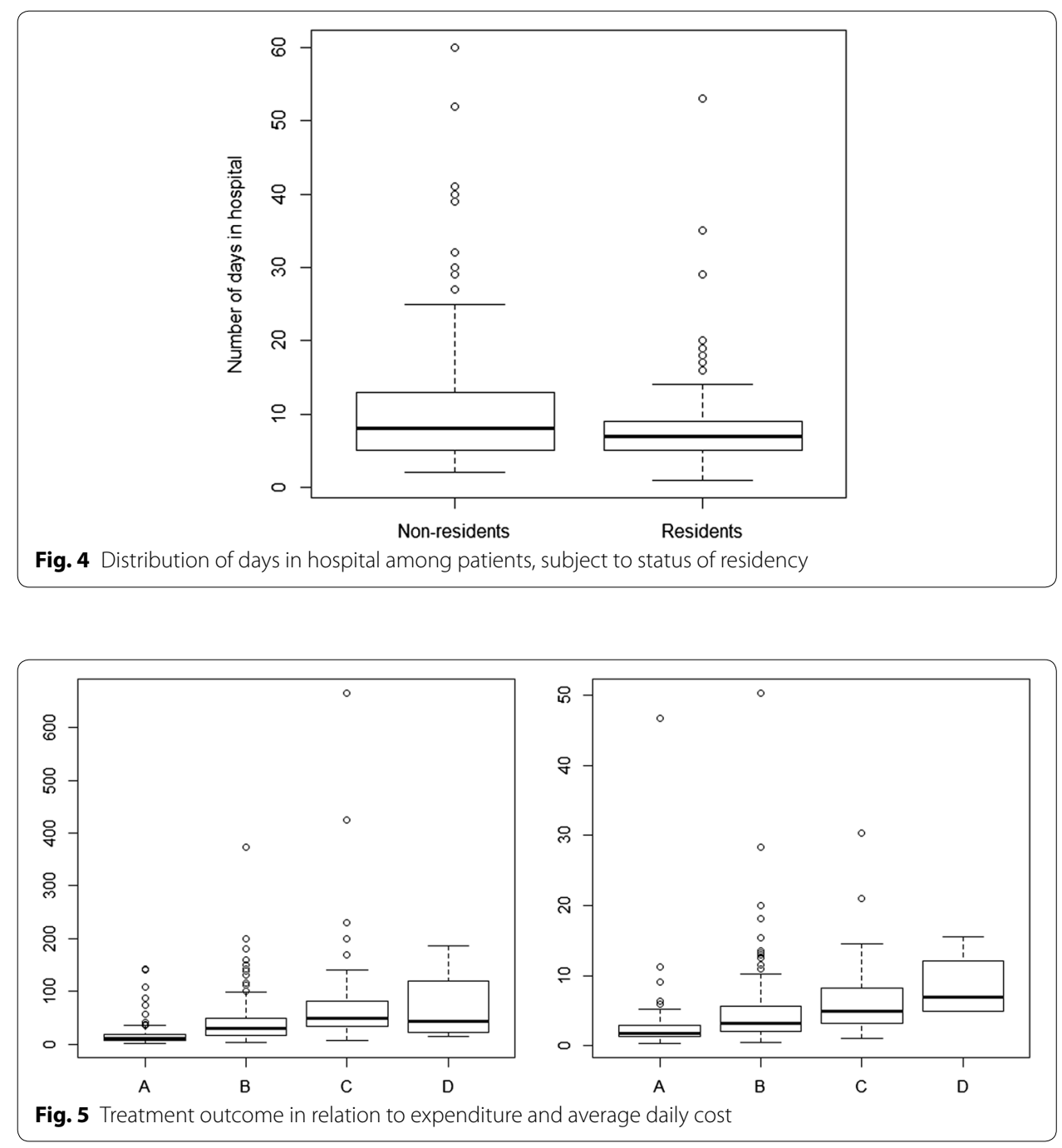

Additional graphs are provided in "Appendix 2" for visual checks on possible relationships among pairs of variables. The data set that is used in subsequent estimations and analyses is provided in sub-tables of Table 10 in the "Appendix 3".

\section{Results: estimated coefficients, functional forms and probabilities}

In what follows, five estimations of joint effects-that likely exacerbate severe impact on probability of destitution-with significant coefficients are reported in separate attempts. In each attempt, coefficients are tabulated, followed by equation forms for stylised facts. Estimated probabilities are computed for the event conditional upon some events specified by the related factors (predictors).

It is noteworthy that in each estimation, no more than two groups of independent categories are used, leading to a limited number of variables entering into specifications. This choice is due to technical requirements for minimum of count value for each cell and the number of cells with count value of less than 5 . In addition, as the survey aims at seeking the effect of changes in individual variables rather than comparing them in more 
complex specifications, a parsimonious specification is preferred in anticipation for better predictive power for computed probabilities.

\section{Joint effects of "Residency" and "Insured" on patients' post-treatment financial distress}

This section starts with the first specification, simple but useful for a general public perception, using sub-table BURDEN1 (from Table 10 in "Appendix 3"). Results are provided in Table 1, with all coefficients being statistically significant, mostly at a conventional level $(p<0.001)$.

Rewriting the above empirical results into the following stylized facts, the first two logits are as follows:

$$
\begin{aligned}
& \ln \left(\frac{\hat{\pi}_{C}}{\hat{\pi}_{A}}\right)=-1.1239+2.2628 \text { NonRes }+0.9652 \text { Uninsured } \\
& \ln \left(\frac{\hat{\pi}_{B}}{\hat{\pi}_{A}}\right)=-0.7349+0.5222 N o n R e s+1.0777 \text { Uninsured }
\end{aligned}
$$

These logits enable us to estimate the probability that a patient falls into debt if that patient is non-resident and uninsured (or medical costs are not eligible for reimbursement under the policy) $\hat{\pi}_{C}$ :

$$
\hat{\pi}_{C}=\frac{e^{-1.1239+2.2628+0.9652}}{1+e^{-1.1239+2.2628+0.9652}+e^{-0.7349+0.5222+1.0777}}=0.7084 .
$$

The probability that a patient falls into some kind of adverse effect (but not indebtedness) and has negligible or no insurance $\hat{\pi}_{B}$ and is a non-resident:

$$
\hat{\pi}_{B}=\frac{e^{-0.9283+1.2128+0.7927}}{1+e^{-0.9462+2.5694+0.7642}+e^{-0.9283+1.2128+0.7927}}=0.2052 .
$$

Consequently, only $8.64 \%(=1-0.7084-0.2052)$ of non-resident patients will not be adversely affected if hospitalised without insurance. A table for distributions of probabilities follows (Table 2).

From probabilities provided in Table 2, it is straightforward to show the contrast of changing probabilities for different burden outcomes depending on status of residency and eligibility for insurance benefits, illustrated in Fig. 6.

Table 1 Estimation results for probability of distress on "residency" and "insured"

\begin{tabular}{llll}
\hline & Intercept & Resident & Insured \\
& $\boldsymbol{\beta}_{\mathbf{0}}$ & No & No \\
& $-1.1239^{* * *}$ & $\boldsymbol{\beta}_{\mathbf{1}}$ & $\boldsymbol{\beta}_{\mathbf{2}}$ \\
\hline Logit $(\mathrm{C} \mid \mathrm{A})$ & {$[0.2738]$} & $2.2628^{* * *}$ & $0.9652^{* * *}$ \\
& $(-4.1046)$ & {$[0.3178]$} & {$[0.3153]$} \\
Logit $(\mathrm{B} \mid \mathrm{A})$ & $-0.7349^{* * *}$ & $(7.1209)$ & $(3.0612)$ \\
& {$[0.2516]$} & $0.5222^{*}$ & $1.0777^{* * *}$ \\
& $(-2.9213)$ & {$[0.3264]$} & {$[0.3349]$} \\
& $(1.5999)$ & $(3.2181)$ \\
\hline
\end{tabular}

Residual deviance: 1.45 on 2 degrees of freedom (df); Log-likelihood: -17.92 on 2 df, Baseline = no financial burden at all; (SE) and $z$ values in parentheses [ ] and ( )

***,**,* Denote coefficients significant at 1,5 and $10 \%$, respectively 
Table 2 Probability distributions financial burden outcome upon status of residency and eligibility for receiving insurance benefits

\begin{tabular}{lllll}
\hline Residency & Eligibility & A & B & C \\
\hline Resident & Yes & 0.5542 & 0.2657 & 0.1801 \\
\multirow{2}{*}{ Non-resident } & No & 0.3065 & 0.4319 & 0.2616 \\
& Yes & 0.2028 & 0.1639 & 0.6333 \\
& No & 0.0864 & 0.2052 & 0.7084 \\
\hline
\end{tabular}

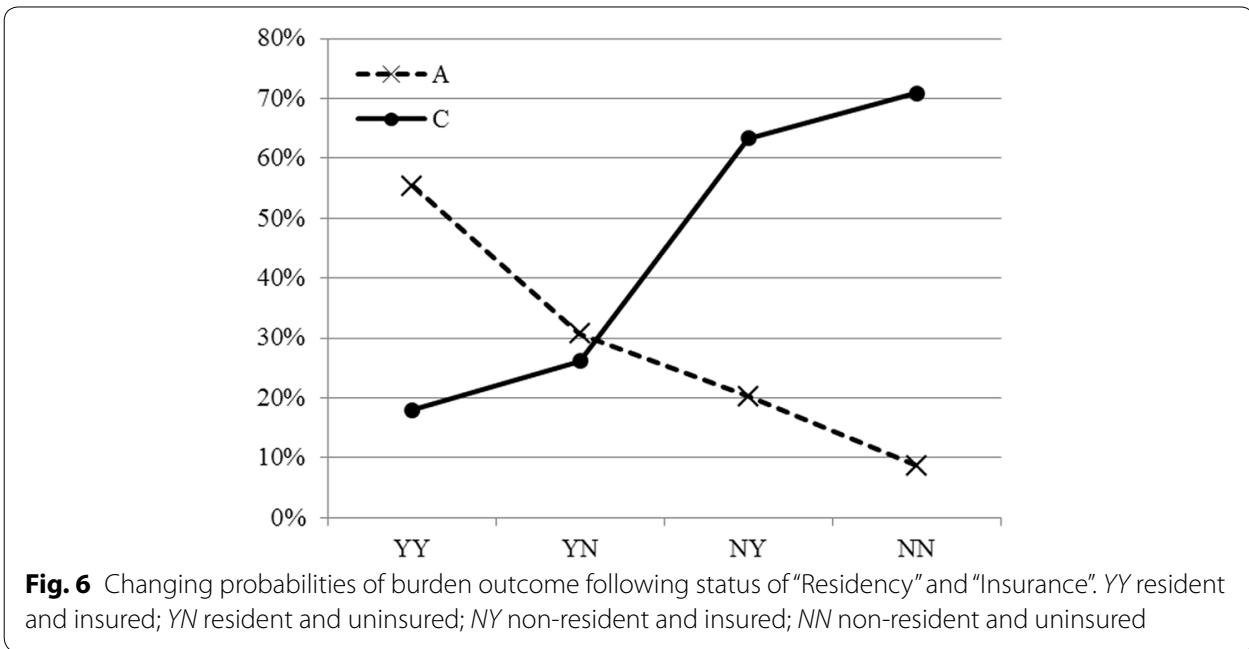

The two lines move in opposite directions. The probability of falling destitute increases when a patient becomes uninsured, but jumps when the patient is non-resident. The highest probability occurs under the joint effect of "uninsured" and "nonresident". On the other hand, the probability of being minimally affected goes down from the best ( $>55 \%$ ) when a patient is both "insured" and "resident" to the worst $(<10 \%)$ when he/she is "uninsured" and "non-resident".

\section{Joint effects of "Insurance benefits" and "Residency" on the probability of distress}

The next estimation models the probability of falling into a specific category of posttreatment "financial position", conditional upon levels of insurance reimbursement and the residency status of the patient and is based on the dataset BURDEN3. Results are provided in Table 3.

The above models the probability of falling into burden category $\mathrm{C}$ (and $\mathrm{B}$ ) versus category A (zero adverse effect of health expenditure), depending on whether a patient is a "non-resident" and "uninsured". The results show a very clear trend. Both burdens of categories C (distressed) and B (partly adversely affected) show significantly negative effects of being a non-resident and having no insurance on patients' probability of becoming indebted. In other words, having no insurance and being a non-resident increases the log-odds of falling into burden type $\mathrm{C}$ or B.

To measure the risk, using results from Table 3, we can take category $\mathrm{C}$ (probability of falling into debt) as an example. The non-residency factor has a much larger (negative) 
Table 3 Modeling probability of financial distress upon "residency" and "insurance benefits"

\begin{tabular}{llllll}
\hline & Intercept & Resident & \multicolumn{2}{l}{ InsL2 } & \\
\cline { 5 - 6 } & & No & Lo & Med & Nil \\
& $\boldsymbol{\beta}_{\mathbf{0}}$ & $\boldsymbol{\beta}_{\mathbf{1}}$ & $\boldsymbol{\beta}_{\mathbf{2}}$ & $\boldsymbol{\beta}_{\mathbf{3}}$ & $\boldsymbol{\beta}_{\mathbf{4}}$ \\
\hline Logit $(C \mid A)$ & $-0.9279^{* * *}$ & $2.3381^{* * *}$ & -0.52045 & -0.6714 & $0.7144^{* *}$ \\
& $(-2.9276)$ & $(7.2249)$ & $(-1.0214)$ & $(-1.4535)$ & $(1.9899)$ \\
Logit (B|A) & $-0.6466^{* *}$ & $0.5808^{*}$ & -0.0468 & -0.5458 & $0.9368^{* *}$ \\
& $(-2.0658)$ & $(1.7572)$ & $(-0.0858)$ & $(-1.0113)$ & $(2.4390)$ \\
\hline
\end{tabular}

Baseline = no financial burden at all; $z$ values in parentheses; Residual deviance: 17.31636 on 6 df; Log-likelihood: -35.4262 with $6 \mathrm{df}$

$* * * * * *$ Denote coefficients significant at 1,5 and $10 \%$ respectively

effect on the probability of a patient becoming indebted than does being uninsured, with the significant coefficient being +2.388 , compared to +0.7144 for being uninsured.

$$
\begin{aligned}
& \ln \left(\frac{\hat{\pi}_{C}}{\hat{\pi}_{A}}\right)=-0.9279+2.3881 \text { NonRes }-0.5204 \text { InsLow }-0.6714 \text { InsMed }+0.7144 \text { InsNil } \\
& \ln \left(\frac{\hat{\pi}_{B}}{\hat{\pi}_{A}}\right)=-0.6466+0.5808 \text { NonRes }-0.0468 \text { InsLow }-0.5458 \text { InsMed }+0.9368 \text { InsNil }
\end{aligned}
$$

It is then possible to compute the probability that a nonresident patient falling into debt having no insurance $\hat{\pi}_{C}$ :

$$
\hat{\pi}_{C}=\frac{e^{-0.9279+2.3881+0.7144}}{1+e^{-0.9279+2.3881+0.7144}+e^{-0.6466+0.5808+0.9368}}=0.6945 .
$$

The probability of a patient without insurance coming from another region and becoming indebted is quite high, almost $70 \%$. In addition, the probability that a nonresident patient falling into some kind of adverse effect-but not indebtedness-having no insurance $\left(\hat{\pi}_{C}\right)$ is much lower, roughly $25 \%$ :

$$
\hat{\pi}_{B}=\frac{e^{-0.6466+0.5808+0.9368}}{1+e^{-0.9279+2.3881+0.7144}+e^{-0.6466+0.5808+0.9368}}=0.2534 .
$$

Only $5.2 \%$ of non-resident patients will be minimally affected if hospitalised without significant insurance benefits, that is $<20 \%$ of total healthcare costs.

\section{The effects of "Health Cost" and "Insurance"}

Next, consider the probabilities of falling into different financial positions (A = Strong, $\mathrm{B}=$ Adversely Affected, or $\mathrm{C}=$ Indebted/Destitute) conditional upon levels of average cost of treatment and insurance reimbursements (sub-table BURDEN4). The baseline category for this regression probability has no negative financial effect; the two reference categories for AvgCost and Insurance Level are LowCost and High reimbursement, respectively.

From Table 4, with the exception of the categorical variable, "Medium Insurance" coverage, being insignificant, most coefficients are statistically significant. Stylised facts from Table 4 are rewritten as: 
Table 4 Modeling categories of financial burden following average cost and insurance levels

\begin{tabular}{|c|c|c|c|c|c|}
\hline & \multirow[t]{3}{*}{$\beta_{0}$} & \multicolumn{2}{|l|}{ AvgCost } & \multicolumn{2}{|c|}{ Insurance level } \\
\hline & & HiCost & MedCost & Med & Neg \\
\hline & & $\beta_{1}$ & $\beta_{2}$ & $\beta_{3}$ & $\beta_{4}$ \\
\hline Logit (C|A) & $\begin{array}{l}-0.9462^{* *} \\
(-2.5037)\end{array}$ & $\begin{array}{l}2.5694^{* * *} \\
(5.1764)\end{array}$ & $\begin{array}{l}1.2158^{* * *} \\
(3.3389)\end{array}$ & $\begin{array}{l}-0.1436 \\
(-0.3233)\end{array}$ & $\begin{array}{l}0.7642^{* *} \\
(2.3656)\end{array}$ \\
\hline Logit (B|A) & $\begin{array}{l}-0.9283^{* *} \\
(-2.3424)\end{array}$ & $\begin{array}{l}1.2128^{* *} \\
(2.2110)\end{array}$ & $\begin{array}{l}0.5020 \\
(1.3182)\end{array}$ & $\begin{array}{l}-0.3656 \\
(-0.6778)\end{array}$ & $\begin{array}{l}0.7927^{* *} \\
(2.1660)\end{array}$ \\
\hline
\end{tabular}

Residual deviance: 5.72 on 8 df; Log-likelihood: -32.19 on $8 \mathrm{df} z$ value in brackets. Baseline category: no financial burden after staying in hospital

******** Denote coefficients significant at 1,5 and $10 \%$, respectively

$$
\begin{aligned}
& \ln \left(\frac{\hat{\pi}_{C}}{\hat{\pi}_{A}}\right)=-0.9462+2.5694 \text { HiCost }+1.2158 \text { MedCost }-0.1436 \text { InsMed }+0.7642 \text { InsNeg } \\
& \ln \left(\frac{\hat{\pi}_{B}}{\hat{\pi}_{A}}\right)=-0.9283+1.2128 \text { HiCost }+0.5020 \text { MedCost-0.3656InsMed }+0.7927 \text { InsNeg }
\end{aligned}
$$

These can be converted into the probability of a patient falling into debt and having negligible insurance benefits while paying high health care cost $\left(\hat{\pi}_{C}\right)$ is as follows:

$$
\hat{\pi}_{C}=\frac{e^{-0.9462+2.5694+0.7642}}{1+e^{-0.9462+2.5694+0.7642}+e^{-0.9283+1.2128+0.7927}}=0.6912 .
$$

The probability that a patient falling into some kind of adverse effect (but not indebtedness) and having negligible or no insurance $\hat{\pi}_{B}$, while paying higher cost of services is:

$$
\hat{\pi}_{B}=\frac{e^{-0.9283+1.2128+0.7927}}{1+e^{-0.9462+2.5694+0.7642}+e^{-0.9283+1.2128+0.7927}}=0.2645 .
$$

Only $4.43 \%$ of patients will not be adversely affected if hospitalised without insurance while paying higher costs. From the results of Tables 3 and 4, it is safe to state that the joint effect of "non-residency" + "uninsured" has a similar impact on risk of destitution (and hardship) as the joint effect of "receiving negligible benefits" + "high costs of health services". To obtain a more interesting finding to see if low insurance benefits are as much of a risk as being uninsured when costs are high, an additional estimation is performed and results are provided in "Appendix 4". Since only $\beta_{5}$ is significant, it is not decisive to compare the two cases.

Next, Table 5 presents the probabilities of falling into different financial positions conditional upon status of insurance ("Insured" and "Uninsured") and Average Cost ("High" and "Medium", and "Low") (sub-table INSURANCE "Appendix 3").

Most coefficients in Table 5, with the exception of "MedCost," are statistically significant. In light of this, financial burdens after treating in hospital are rewritten as:

$$
\ln \left(\frac{\hat{\pi}_{C}}{\hat{\pi}_{A}}\right)=-1.0346+2.6368 \text { HiCost }+1.2653 \text { MedCost }+1.1261 \text { Uninsured }
$$


Table 5 Modelling categories of financial burden following average cost and insurance status

\begin{tabular}{lllll}
\hline & $\boldsymbol{\beta}_{\mathbf{0}}$ & AvgCost & & Insurance \\
\cline { 3 - 5 } & & High cost & Med. cost & Uninsured \\
& & $\boldsymbol{\beta}_{\mathbf{1}}$ & $\boldsymbol{\beta}_{\mathbf{2}}$ & $\boldsymbol{\beta}_{\mathbf{3}}$ \\
\hline Logit (C|A) & $-1.0346^{* *}$ & $2.6368^{* * *}$ & $1.2653^{* * *}$ & $1.1261^{* * *}$ \\
& {$[-3.070]$} & {$[5.279]$} & {$[3.459]$} & {$[3.689]$} \\
Logit (B|A) & $-1.0328^{* *}$ & $1.2865^{*}$ & 0.5621 & $1.1223^{* * *}$ \\
& {$[-3.018]$} & {$[2.342]$} & {$[1.477]$} & {$[3.316]$} \\
\hline
\end{tabular}

Residual deviance: 10.91 on 4 df; Log-likelihood: -28.18 on 4 df z value in brackets. Baseline category: no financial burden after staying in hospital

******** Denote coefficients significant at 1,5 and $10 \%$, respectively

Table 6 Summary of probabilities of destitution on "Insurance" and "High Cost"

\begin{tabular}{lllllll}
\hline $\begin{array}{l}\text { Burden } \\
\text { Avg. cost }\end{array}$ & $\begin{array}{l}\text { A } \\
\text { Uninsured }\end{array}$ & B & C & $\begin{array}{l}\text { A } \\
\text { Insured }\end{array}$ & B & C \\
\hline High & 0.0493 & 0.1954 & 0.7553 & 0.1379 & 0.1777 & 0.6844 \\
Low & 0.3135 & 0.3429 & 0.3436 & 0.5843 & 0.2080 & 0.2077 \\
Medium & 0.1470 & 0.2821 & 0.5709 & 0.3467 & 0.2166 & 0.4367 \\
\hline
\end{tabular}

$$
\ln \left(\frac{\hat{\pi}_{B}}{\hat{\pi}_{A}}\right)=-1.0328+1.2865 \text { HiCost }+0.5621 \text { MedCost }+1.1223 \text { Uninsured }
$$

The conditional probability of patients who are falling into indebtedness (category C) while paying high costs and having no insurance is as follows:

$$
\hat{\pi}_{C}=\frac{e^{-1.0346+2.6368+1.1261}}{1+e^{-1.0346+2.6368+1.1261}+e^{-1.0328+1.2865+1.1223}}=0.7553 .
$$

The above results suggest that when uninsured patients have to pay higher costs their probability of going destitute becomes $\sim 75.5 \%$, significantly higher than the probability of $69.1 \%$ as derived from Table 4 . Furthermore, the joint effects of paying high cost and being (in) eligible for insurance benefits on probabilities of different burden outcomes, computed from Table 5, are shown by the distributions in Table 6 .

To visualize the effects of changes in level of health costs and eligibility for receiving insurance benefits, Fig. 7 may be useful. Regarding best outcome (A), when facing high costs of treatment, the probability for the "uninsured" falls by almost 9 percentage point from that of the "insured" (13.8-4.9). But for the worst outcome (C), the gap is narrower: (75.5-68.4) $=7$ percentage point. Comparatively, the effect of change in A, when a patient moves from "insured" to "uninsured", is much stronger (9/13.8) than in $\mathrm{C}(7 / 75.5)$. That is to say the negative effect of being uninsured is rather 'stable' in determining the probabilities of going destitute.

The slopes of two lines in Fig. 7 also indicate that the "uninsured" is generally disadvantageous compared to the insured, with respect to the distinct outcomes $\mathrm{A}$ and $\mathrm{C}$ that the survey wants to observe. For the moderately affected cases (category B), the difference appears to be quite negiligible. 


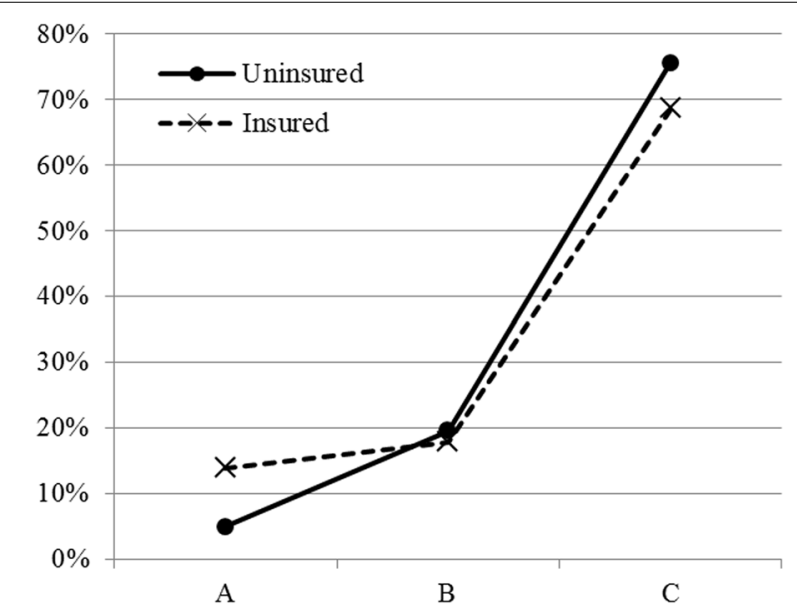

Fig. 7 Changing probabilities of category A and C burden outcome following high costs and eligibility of insurance

\section{Treatment outcome, health care cost and severity of illness}

The next analysis focuses on the probabilities of "Treatment Outcome" for patients, conditional upon average cost of services and severity of illness, employing the END2 subset from Table 10 in the "Appendix 3". Estimated coefficients are provided in Table 7, following which, most coefficients are highly significant. Table 7 results are rewritten in equation forms as follows:

$$
\begin{aligned}
& \ln \left(\frac{\hat{\pi}_{C}}{\hat{\pi}_{A}}\right)=-4.5965+2.3444 \text { Bad }+4.1486 \text { Emergency }+3.2568 \text { High Cost }+1.2881 \text { MedCost } \\
& \ln \left(\frac{\hat{\pi}_{B}}{\hat{\pi}_{A}}\right)=-1.1231+1.1452 \text { Bad }+1.1074 \text { Emergency }+2.4116 \text { HighCost }+1.08 \text { MedCost }
\end{aligned}
$$

The results show that the probability that a patient quits while in a condition of "serious illness" and anticipating expensive treatments $\left(\hat{\pi}_{C}\right)$ is quite high, $58 \%$ :

$$
\hat{\pi}_{C}=\frac{e^{-4.5965+4.1486+3.2568}}{1+e^{-4.5965+4.1486+3.2568}+e^{-1.1231+1.1074+2.4116}}=0.5805 .
$$

Likewise, the probability that a patient can only be partially cured $\hat{\pi}_{B}$ while paying high

\begin{tabular}{|c|c|c|c|c|c|}
\hline & \multirow[t]{2}{*}{ Intercept } & \multicolumn{2}{|l|}{ Illness } & \multicolumn{2}{|c|}{ Average cost of services } \\
\hline & & Bad & Emergency & High & Medium \\
\hline & $\beta_{0}$ & $\beta_{1}$ & $\beta_{2}$ & $\beta_{3}$ & $\beta_{4}$ \\
\hline Logit $(C \mid A)$ & $\begin{array}{l}-4.5965^{* * *} \\
(-4.9401)\end{array}$ & $\begin{array}{l}2.3444^{* * *} \\
(2.9511)\end{array}$ & $\begin{array}{l}4.1486^{* * *} \\
(5.0950)\end{array}$ & $\begin{array}{l}3.2568^{* * *} \\
(4.0096)\end{array}$ & $\begin{array}{l}1.2881^{*} \\
(1.8532)\end{array}$ \\
\hline Logit (B|A) & $\begin{array}{l}-1.1231^{* * *} \\
(-3.5675)\end{array}$ & $\begin{array}{l}1.1452^{* * *} \\
(3.8388)\end{array}$ & $\begin{array}{l}1.1074^{* * *} \\
(2.6608)\end{array}$ & $\begin{array}{l}2.4116^{* * *} \\
(4.6114)\end{array}$ & $\begin{array}{l}1.0800^{* * *} \\
(3.2739)\end{array}$ \\
\hline
\end{tabular}
cost of services is also substantial, over $38 \%$ :

Table 7 Modeling "Treatment Outcome" following cost levels and illness

Residual deviance: 14.36 on $8 \mathrm{df}$; Log-likelihood: -32.17 on $8 \mathrm{df}$ z value in brackets. Baseline category: complete recovery after treatments

***:***** Denote coefficients significant at 1,5 and $10 \%$, respectively 


$$
\hat{\pi}_{B}=\frac{e^{-1.1231+1.1074+2.4116}}{1+e^{-4.5965+4.1486+3.2568}+e^{-1.1231+1.1074+2.4116}}=0.3845
$$

In the estimated $\hat{\pi}_{C}$, both conditions of illness and high costs have a large impact on increasing the risk of early quitting. However, in case of $\hat{\pi}_{B}$ costliness of treatment appears to be the more determining factor.

These two high probabilities lead to the fact that the probability of full recovery for a patient hospitalised with an emergency, anticipating higher costs of treatment, is very low, just $3.5 \%$.

\section{On the sensitive issue of "extra thank-you money" OOP}

Finally, estimation results are reported based on data provided in the ENV2 sub-table, which model the probability of a patient paying high or medium "extra thank-you money" conditional upon income ranks and/or severity of illness. The baseline category is "paying negligible thank-you" for the response variable. For "Ill2" the reference category is "light sickness" and for "Income Rank", the reference is "Medium".

Clearly, the estimated results in Table 8 show that both income ranks of the category Low (of "Income Rank") and both Bad and Emergency (of "Illness") jointly reduce the probability of patients paying "thank-you money" from medium to high level. That is, these lower-income patients, when faced with serious illness or emergency, are less likely to be able to afford significant "Extra thank-you money" OOP payments.

$$
\begin{aligned}
& \ln \left(\frac{\hat{\pi}_{\text {HiPay }}}{\hat{\pi}_{\text {NegPay }}}\right)=+0.5079-1.7684 \text { Bad }-1.4804 \text { Emerg }-0.5079 \text { HiInc }-0.9134 \text { LowInc } \\
& \ln \left(\frac{\hat{\pi}_{\text {MedPay }}}{\hat{\pi}_{\text {NegPay }}}\right)=+0.6104-1.0751 \text { Bad }-1.4605 \text { Emerg }-0.9899 \text { HiInc }-1.0804 \text { LowInc }
\end{aligned}
$$

Still, the poor who have an "emergency" are willing to make an expensive "thank-you" OOP payment with a probability $\left(\hat{\pi}_{\text {HiPay }}\right)$ of $7.8 \%$ following the above estimation:

\begin{tabular}{|c|c|c|c|c|c|}
\hline & \multirow[t]{3}{*}{$\beta_{0}$} & \multicolumn{2}{|l|}{ Illness } & \multicolumn{2}{|c|}{ Income rank } \\
\hline & & Bad & Emergency & High & Low \\
\hline & & $\beta_{1}$ & $\beta_{2}$ & $\beta_{3}$ & $\beta_{4}$ \\
\hline Logit (HiPay|Neg) & $\begin{array}{l}0.5079 \\
{[0.5813]} \\
(0.8737)\end{array}$ & $\begin{array}{l}-1.7684^{* * *} \\
{[0.4560]} \\
(-3.8778)\end{array}$ & $\begin{array}{l}-1.4804^{* * *} \\
{[0.4851]} \\
(-3.0520)\end{array}$ & $\begin{array}{l}-0.5079 \\
{[0.6657]} \\
(-0.7629)\end{array}$ & $\begin{array}{l}-0.9134^{*} \\
{[0.4835]} \\
(-1.8891)\end{array}$ \\
\hline Logit (MedPay|Neg) & $\begin{array}{l}0.6104 \\
{[0.5128]} \\
(1.1902)\end{array}$ & $\begin{array}{l}-1.0751^{* * *} \\
0.41346 \\
(-2.6003)\end{array}$ & $\begin{array}{l}-1.4605^{* * *} \\
{[0.4880]} \\
(-2.9930)\end{array}$ & $\begin{array}{l}-0.9899 \\
{[0.6265]} \\
(-1.5799)\end{array}$ & $\begin{array}{l}-1.0804^{* * *} \\
{[0.3933]} \\
(-2.7471)\end{array}$ \\
\hline
\end{tabular}

$$
\hat{\pi}_{\text {HiPay }}=\frac{e^{-1.4804-0.9134}}{1+e^{-1.4804-0.9134}+e^{-1.4605-1.0804}}=0.0780 .
$$

Table 8 Modeling "Extra thank-you money" against "IIIness" and "Income Rank"

Residual deviance: 3.81 on $2 \mathrm{df}$; Log-likelihood: -24.11 on $2 \mathrm{df}$

[SE] in square bracket and (z value) in parentheses. Baseline category: pay negligible "extra envelop" amount

******* Denote coefficients significant at 1,5 and $10 \%$, respectively 
Likewise, $\hat{\pi}_{\text {MedPay }}$ is estimated at $\sim 6.7 \%$ :

$$
\hat{\pi}_{\text {MedPay }}=\frac{e^{-1.4605-1.0804}}{1+e^{-1.4804-0.9134}+e^{-1.4605-1.0804}}=0.0673 .
$$

After all, it is with a high probability, $85.46 \%$, that low-income patients with an emergency can only afford a negligible amount of "extra thank-you" OOP payment.

\section{Discussion and concluding remarks}

The attempts to model the risk of destitution among patients suggest that that key variables are crucial. The following discusses those relationships, concluding remarks, and implications for policy making and the efficient use of insurance by patients.

Clearly, being hospitalized without insurance represents a high risk of going destitute for all in-patients. The chance of being minimally affected in terms of financial situation after expensive in-patient improves significantly for insured patients. The practice of travelling from rural areas to large cities, in hopes of better healthcare services also adds to the probability of becoming destitute. This is because of unexpected costs such as informational asymmetry, and travel costs. It is highly probable that for every three uninsured and nonresident hospitalised patients, two will face serious financial hardship or destitution. Further, results suggest that for every two patients hospitalised with a serious illness requiring costly treatment, it is highly likely that at least one would risk going destitute. Although researchers would say the risk is high, an important question is "how high is high" to provide the public and policy-makers with more insightful answers. These probabilities suggest that Vietnamese patients are more vulnerable to the risk of destitution than previously thought.

The finding that low-income patients with emergency or serious illness are less likely to give high amounts of "extra thank-you money" suggests that the amount is a small proportion of treatment cost. "Thank-you money" may be a cultural norm showing patients' respect for people taking care of their health or even saving their lives. In light of this, physicians should accept this respectable gesture. Moreover, as the probability of full recovery is low, patients-especially those are hospitalized with an emergency-are more willing to make good relationships with hospital staff members since they are concerned about a future hospital visit.

Other forms of OOP expenses may include "payment" for access to governmentfunded programs, such as treatments with advanced equipment and/or expensive drugs. Selvaraj (2010) reveals that OOP spending has increased in India due to a substantial surge of drug prices over the years. Such practices may make the programs-i.e., health care insurance-end up with a narrower focus than original mandated, even widening the gaps between groups of patients in different levels of income. In addition to coverage expansion, further health care reforms should improve the equality of distribution of benefits (Chen et al. 2014, 2015). Further, it could alleviate the moral hazards of beneficiaries with high health insurance coverage levels (Kim et al. 2015). Understanding these challenges calls for further research.

Given the fact that only $5.2 \%$ of non-resident patients will be minimally affected if they are hospitalised without significant insurance benefits, popular bypassing may exacerbate rural-urban disparities. Indeed, it has happened in Malaysia (Loganathan et al. 2015) and China (Hu et al. 2008), where most medical resources are allocated to 
urban areas. In such a circumstance, inequalities are hardly narrowed by expansions of health insurance coverage and service utilization (Fu et al. 2014).

In addition to travelling and accommodation burdens, bypassing patients face asymmetric information. The patients, particularly who are poor and come from rural areas, often make OOP payments to access allegedly better medical treatment even if they risk falling into severe indebtedness. Their "bargaining power" is weak as they usually make payment without asking for "deliverables". Limited information, which is collected from small group of patients whose situations are similar, may lead the uninsured to overpay but also to not have access to medicines being abundant in Vietnam (Mao et al. 2015). The patients may make OOP payments for special and advanced medicines without knowing whether those medicines are needed or likely to help. Their judgments are almost based on price and scarcity of the medicines so they believe the more expensive and rare the medicines are, the more effective they must be. Such misleading perception may add unnecessary, but possibly expensive, expenses to the treatment costs. The expenses, in turn, raise the probability of being destitute.

For health services providers, including hospitals, the results suggest that their future depends on the payment servicing capacity, and the risk is high for patients when costs are high. The risk is exacerbated if for some reasons (in fact, the reasons are countless) a patient is not eligible for a substantial portion of insurance package that he/she is entitled to, in principle, $\sim 70 \%$. Leaving patients destitute after treatments, due to high costs and inadequately covered by insurance, could damage the hospital's reputation and future. This implies a more proactive coordination between hospitals and health insurance authorities in alleviating the obstacles to eligible rights of the patients.

As the amended Law on Health Insurance comes into effect, the ambitious plan of aiming at $100 \%$ UC and all Vietnamese having health insurance faces a dilemma. While the current statistics show that roughly $60 \%$ of Vietnamese hold UC, the majority of insured patients could hardly be financed adequately by insurance, see Fig. 2a, b. One could only guess what would happen if this current level of $60 \%$ insured increased to $100 \%$; it is likely that the actual coverage range would go down. If this decrease would lead to higher rate of "negligible insurance", the computed probabilities would enable us to predict that the probability of falling into destitution may even rise. In fact, before the introduction of the new law, tension already ran high at times in the 2013-2014 period upon news of a possible collapse of the Vietnam Health Insurance Fund, causing deep concerns in society. This says that, without an appropriate evidence-based policy making process, an idea that is nice initially may eventually end up penalising the poor.

\section{Statement of research ethics:}

I ensure the basic principles of ethical research as stated below:

- Beneficence: As a researcher I strive to ensure that my work makes a positive contribution to the welfare of those affected by it.

- Non-malfeasance: I endeavour to ensure that the research work does not cause harm to any sectors of society and, in particular, to participants.

- Justice: The benefits and risks associated with this study should be well assessed in advance and both should be equitably distributed throughout society. 
- Autonomy of subjects: The research respects and protects the rights and dignity of participants.

As the research involves primary data collection, in the form of interview surveys, I have been fully compliant with all legal requirements regarding the collection, storage, handling, processing and analysis of data. I guarantee that I have conformed to the highest standards of:

- Veracity: Participants in sample surveys and data collection exercises are given full and accurate information regarding issues such as the background, nature, purpose, and outputs of the research.

- Informed consent: Participants in sample surveys and related data collection exercises were given sufficient details on the research in question as to allow them to make an informed decision to participate or otherwise in a research study.

- Protection of vulnerable groups: I am particularly conscious of my obligations to safeguard the interests of vulnerable or potentially 'at risk' groups who may be involved in my research.

- Privacy: Participants in data collection exercises have the right not only to agree to participate in the research but also to decide to withdraw from the research at any time.

- Confidentiality: The information provided by participants is treated as confidential and used for research purposes only. Micro-level information will not be disclosed in any fashion to third parties, which would allow it to be associated with an identifiable individual.

- Minimising risk: Participants in the research will not be put under undue or unnecessary risk as a result of their participation.

- Research outputs: I am committed to putting the results of this research into the public domain (always on an anonymous basis) with a view to transparency, scrutiny and peer review. Where feasible, I am committed to depositing anonymous primary data collected in the course of research into a publicly accessible data archive.

\section{Additional files}

Additional file 1: Analysis of patients survey 2014 (330 cases).

Additional file 2: Data tables for estimations.

Additional file 3: Research ethics compliance.

\section{Acknowledgements}

I would like to thank my friends and colleagues who have provided assistance and support for this research: Nghiem Phu Kien Cuong (Viet Duc Hospital, Hanoi), Luong Minh Ha, Tran Tri Dung and Le Thi Nga (DHVP Research), Nguyen Pham Muoi (Wall Street Journal), and Dau Thuy Ha (OCD Consultants). My work also benefits from discussions with Nancy K. Napier and Kirk Smith (Boise State University, ID, USA), and Dolly Samson (Stamford International University, Bangkok). I am also indebted to hundreds of patients and their family members for their willingness to answer deep, and often timeconsuming, interview questions; without their information this study would not be possible.

Compliance with ethical guidelines

Competing interests

The author declares that he has no competing interests.

\section{Appendix 1}

See Table 9. 

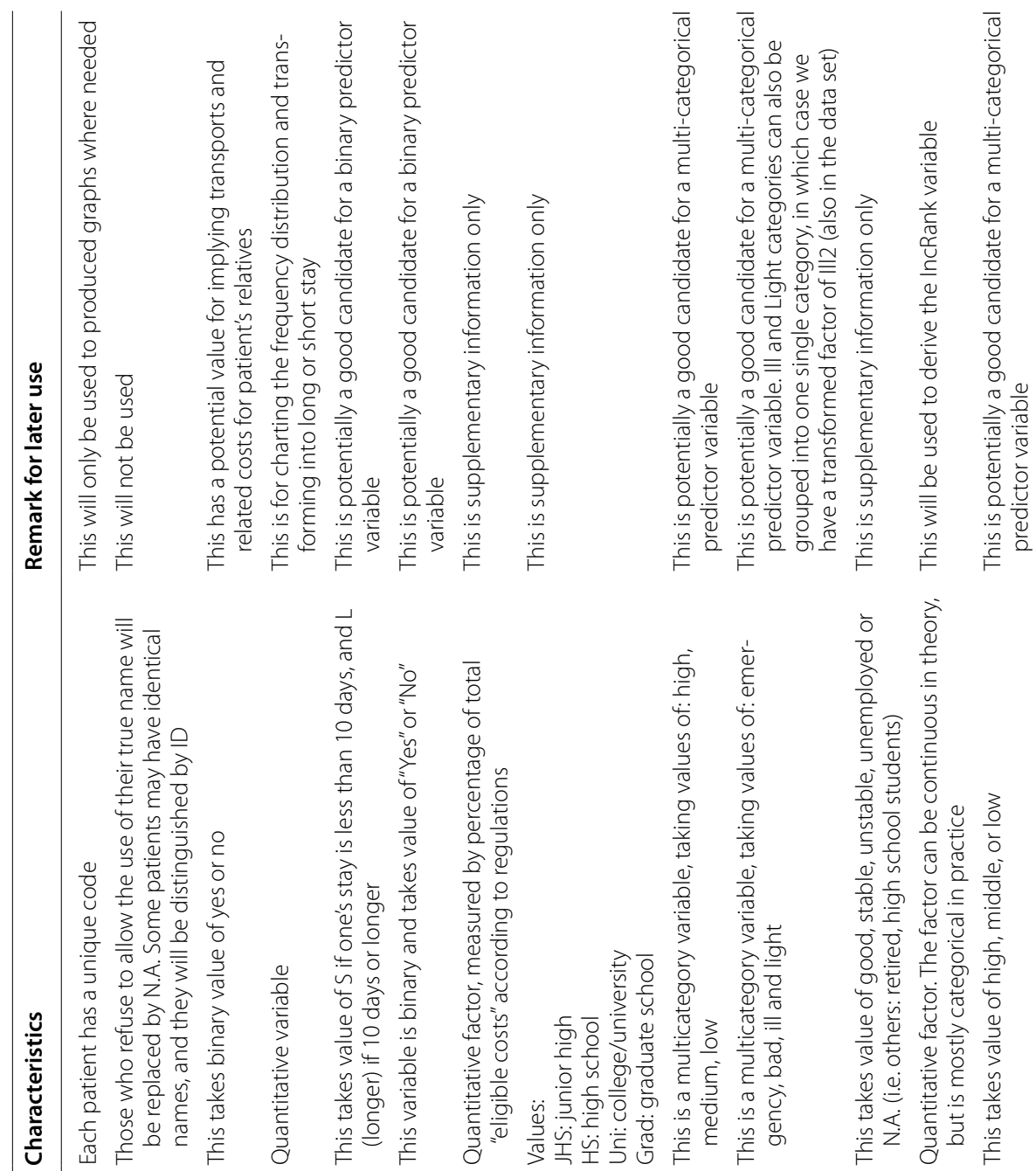

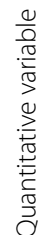

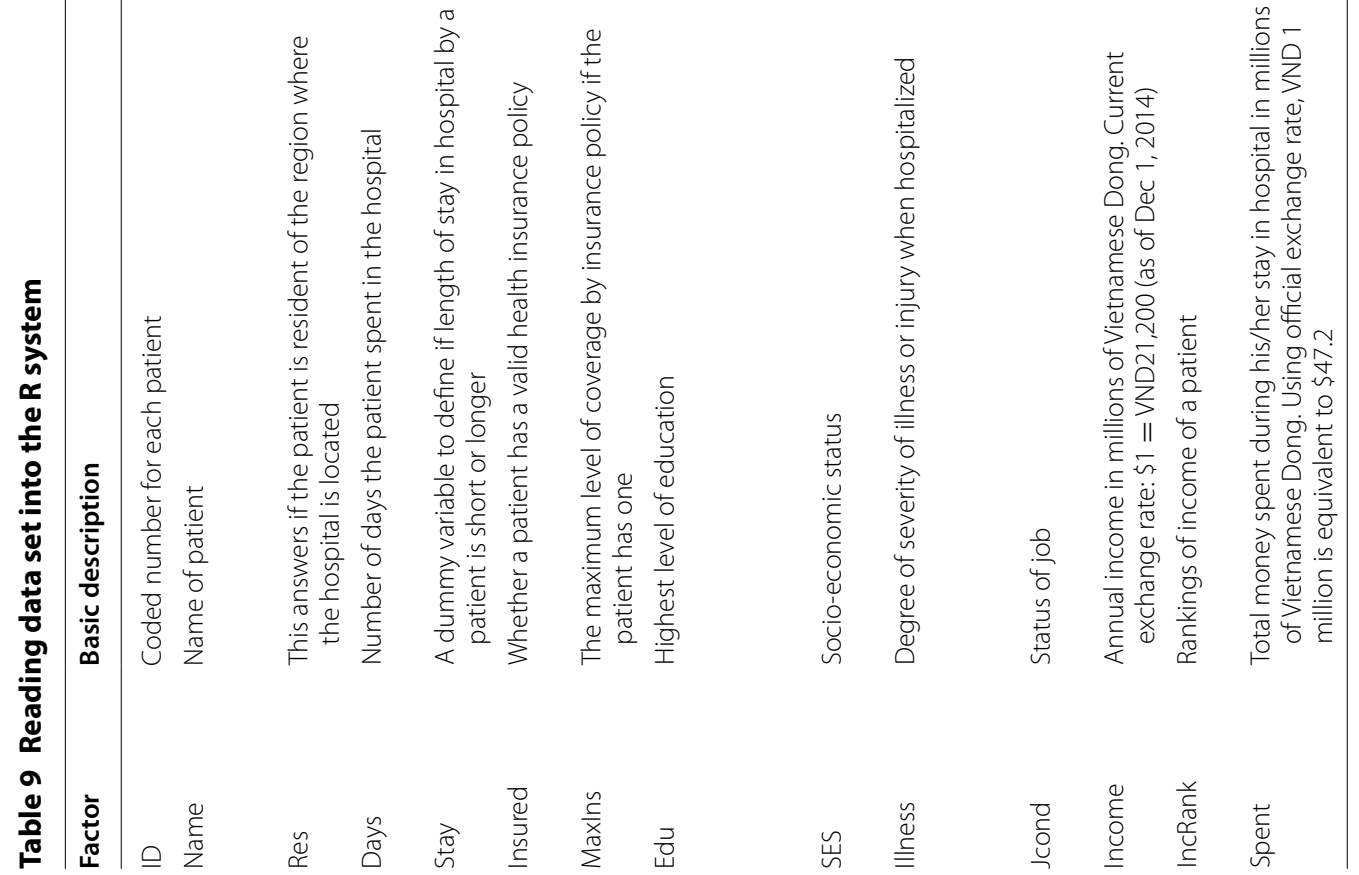




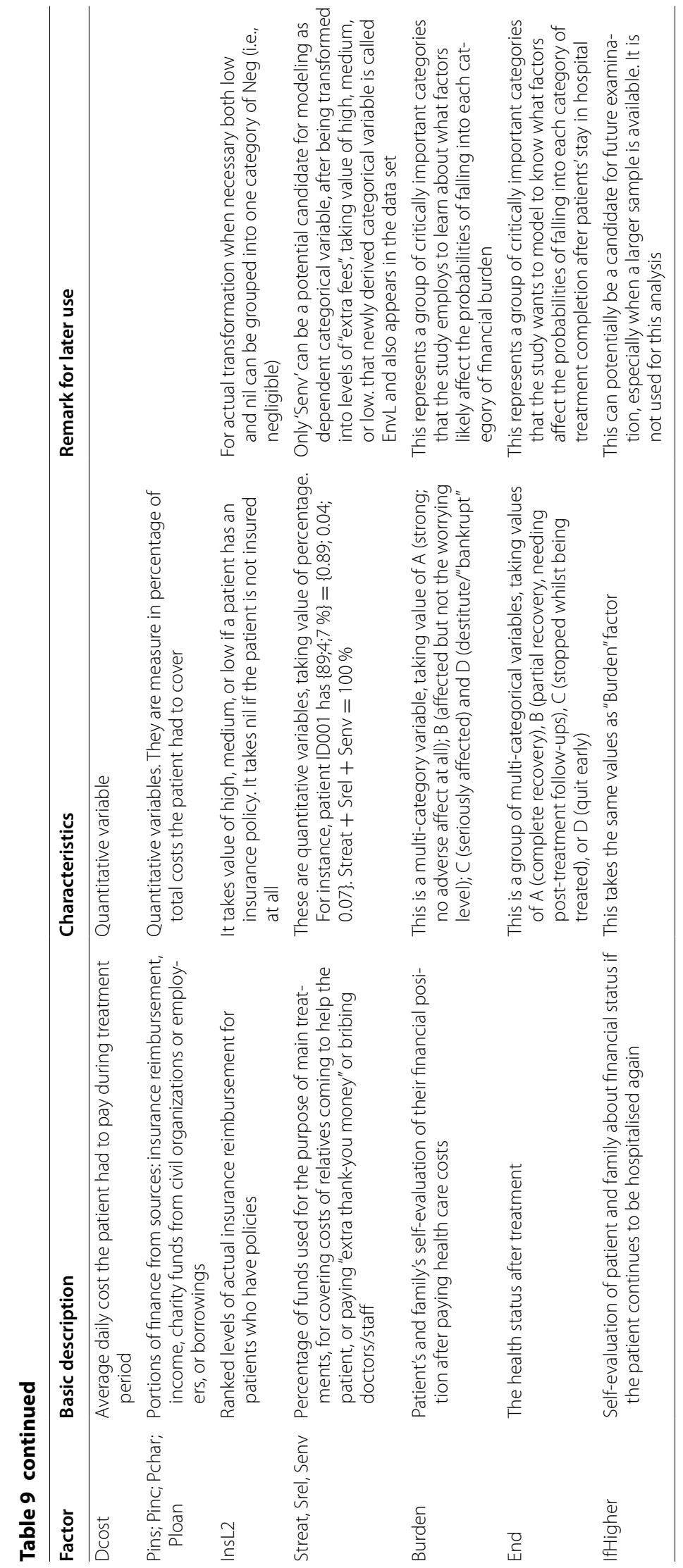




\section{Appendix 2}

See Figs. 8 and 9; Additional file 3.

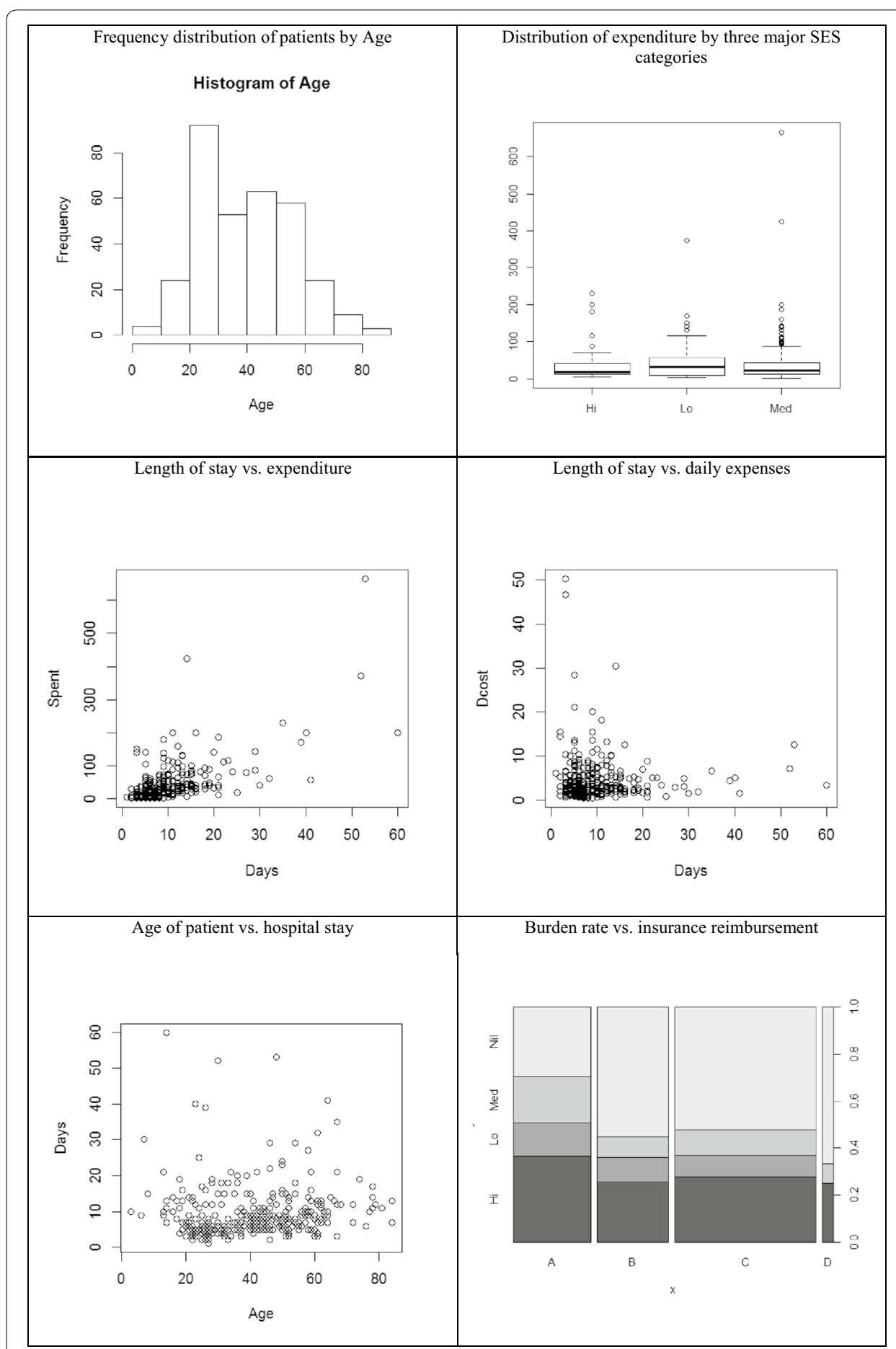

Fig. 8 Supplementary graphs for visualisation of data set 


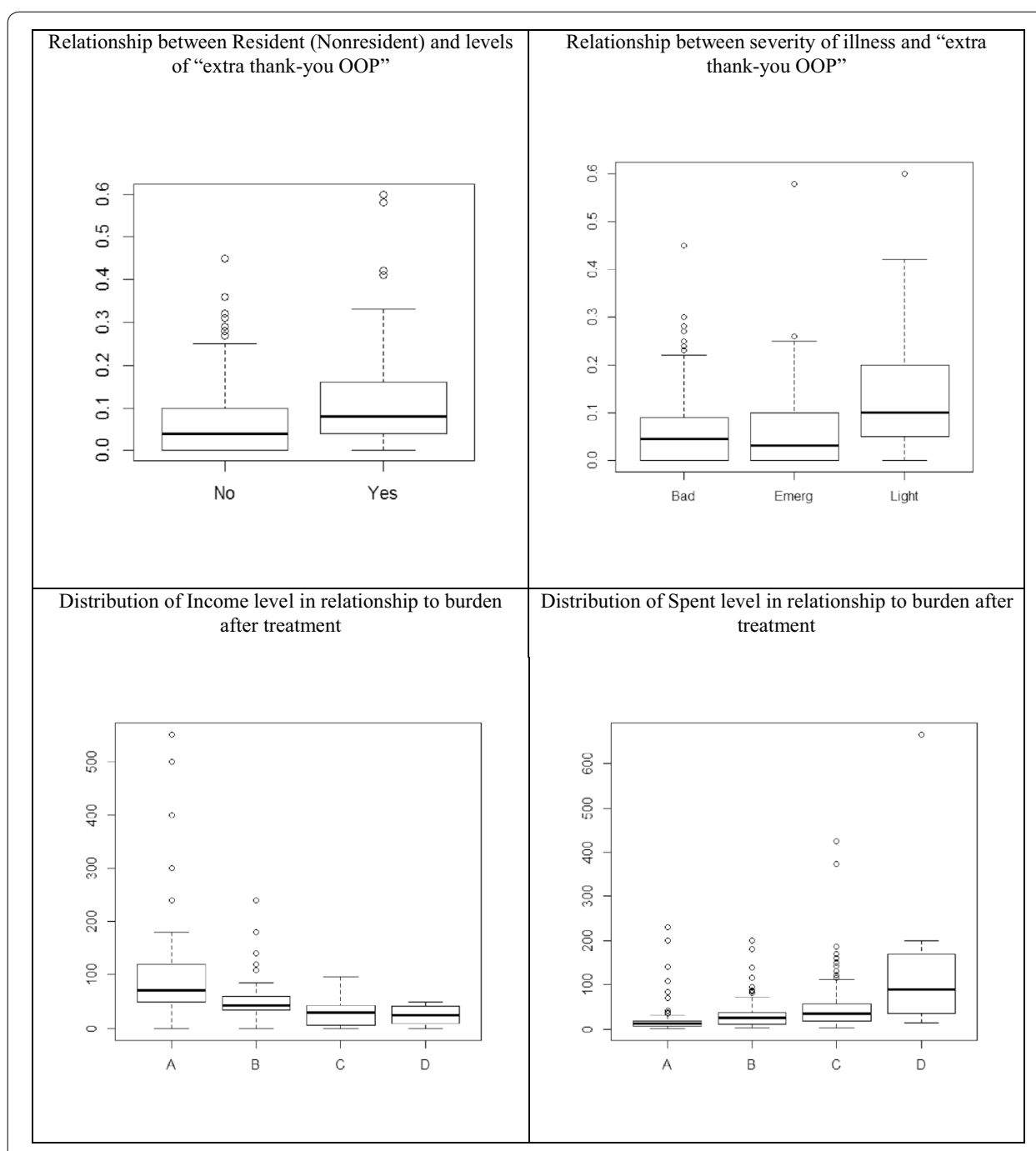

Fig. 9 Supplementary graphs for visualisation of data set

\section{Appendix 3: The data set and contingency tables}

The data set used in this analysis is structured into 5 contingency tables (called "subtables") containing categorical response and predictor variables produced for relevant estimations, and corresponding count values. Each sub-table is given a name for easier reference in upcoming discussions (see Table 10).

\section{Appendix 4}

See Table 11. 
Table 10 The data set and contingency tables

\#\# BURDEN3

InsL2 Res A B C

Hi No 5941

Hi Yes 26115

Med No 11116

Med Yes 662

Nil No 112472

Nil Yes 141917

Lo No 4513

Lo Yes 831

\section{\#\# BURDEN1}

\#\#

Resident Insured A B C

Nonres No 92371

Nonres Yes 221671

Res No 141814

Res Yes 402111

\#\# ENV2

III2 IncRank Hi Med Nil

Bad HM 71317

Bad L 81786

Emerg HM 129

Emerg L 9847

Light Hi 191319

Light L 161524

\#\#INSLEVEL

InsL2 AvgCost A B C

$\mathrm{Hi} \mathrm{Hi} 3422$

Hi Lo 1334

Hi Med 151320

Lo Hi 026

Lo Lo 410

Lo Med 858

Med Hi 118

Med Lo 832

Med Med 838

Nil Hi 4718

Nil Lo 5910

Nil Med 162761
\#\# BURDEN4

AvgCost InsL2 A B C

HiCost Hi 3422

HiCost Med 118

HiCost Neg 4924

MedCost Hi 151319

MedCost Med 838

MedCost Neg 243269

LowCost Hi 1334

LowCost Med 832

LowCost Neg 91010

\#\# END2

III2 AvgCost A B C

Bad Hi 3336

Bad Med 17599

Bad Lo 1281

Emerg Hi 0814

Emerg Med 62417

Emerg Lo 511

Light Hi 381

Light Med 34260

Light Lo 21121

\#\#INSURANCE

Insured AvgCost A B C

No Hi 4717

No Lo 4910

No Med 152558

Yes Hi 4737

Yes Lo 2676

Yes Med 322339

Checked against the data structure and requirement for minimal count value of a cell and all cells, particularly for polytomous logistic regression, the empirical data presented in these sub-tables are satisfactory and ready for estimations

Table 11 Test results on relationship between "Average Cost" and "Insurance Levels": Reported coefficients are estimated using the data of contingency table INSLEVEL (see "Appendix 3")

\begin{tabular}{|c|c|c|c|c|c|c|}
\hline \multirow{3}{*}{$\begin{array}{l}\text { Modelling categories of financial burden } \\
\text { following Average Cost and Insurance } \\
\text { Level }\end{array}$} & \multirow[t]{3}{*}{$\beta_{0}$} & \multicolumn{2}{|l|}{ AvgCost } & \multicolumn{3}{|c|}{ Insurance level } \\
\hline & & HiCost & MedCost & Med & Low & Nil \\
\hline & & $\beta_{1}$ & $\beta_{2}$ & $\beta_{3}$ & $\beta_{4}$ & $\beta_{5}$ \\
\hline Logit (C|A) & $\begin{array}{l}-0.9515^{*} \\
(-2.505)\end{array}$ & $\begin{array}{l}2.631^{* * *} \\
(5.255)\end{array}$ & $\begin{array}{l}1.2503^{* * *} \\
(3.397)\end{array}$ & $\begin{array}{l}-0.1669 \\
(-0.375)\end{array}$ & $\begin{array}{l}-0.2665 \\
(-0.549)\end{array}$ & $\begin{array}{l}1.0133^{* *} \\
(2.933)\end{array}$ \\
\hline Logit (B|A) & $\begin{array}{l}-0.9454^{*} \\
(-2.375)\end{array}$ & $\begin{array}{l}1.2636^{*} \\
(2.292)\end{array}$ & $\begin{array}{l}0.5224 \\
(1.361)\end{array}$ & $\begin{array}{l}-0.3641 \\
(-0.674)\end{array}$ & $\begin{array}{l}0.0053 \\
(0.010)\end{array}$ & $\begin{array}{l}1.0263^{* *} \\
(2.642)\end{array}$ \\
\hline
\end{tabular}

Residual deviance: 13.64 on 12 df; Log-likelihood: -40.81 on $12 \mathrm{df}$ z value in brackets. Baseline category: no financial burden after staying in hospital

******** Denote coefficients significant at 1,5 and $10 \%$, respectively 
Received: 18 December 2014 Accepted: 27 August 2015

Published online: 21 September 2015

\section{References}

Agresti A (2002) Categorical data analysis. Wiley, Hoboken

Bloom G (1997) Primary health care meets the market: lessons from China and Vietnam. IDS Working Paper 53, University of Sussex, UK

Bronstein JM, Morrisey MA (1991) Bypassing rural hospitals for obstetrics care. J Health Polit Policy Law 16(1):87-118

Buczko W (1994) Bypassing of local hospitals by rural medicare beneficiaries. J Rural Health 10(4):237-246

Chaudhuri A, Roy K (2008) Changes in out-of-pocket payments for healthcare in Vietnam and its impact on equity in payments, 1992-2002. Health Policy 88(1):38-48

Chen M, Zhao Y, Si L (2014) Who pays for health care in China? The case of Heilongjiang Province. PLoS One 9(10):e108867. doi:10.1371/journal.pone.0108867

Chen M, Fang G, Wang L, Wang Z, Zhao Y, Si L (2015) Who benefits from government healthcare subsidies? An assessment of the equity of healthcare benefits distribution in China. PLoS One 10(3):e0119840. doi:10.1371/journal. pone.0119840

Daemmrich A (2013) The political economy of healthcare reform in China: negotiating public and private. SpringerPlus 2:488. doi:10.1186/2193-1801-2-448

Dan Tri Online (2014). Hon 47 trieu dong den nguoi me tre bi u nao [More than 47 million dong for young mom with brain tumor]. http://dantri.com.vn/tam-long-nhan-ai/trao-hon-47-trieu-dong-den-nguoi-me-tre-bi-u-nao-5-landanh-roi-con-1008768.htm. Accessed 17 Dec 2014

Dang BH, Bales S, Nguyen KP, Jiaying C, Segall M, Lucas H (2006) Ensuring health care for the rural poor in Vietnam and China: a state or a market approach?. Medical Publishing House, Hanoi

Dao HT, Waters H, Le QV (2008) User fees and health service utilization in Vietnam: how to protect the poor? Public Health 122(10):1068-1078

Ekman B, Liem NT, Duc HA, Axelson H (2008) Health insurance reform in Vietnam: a review of recent developments and future challenges. Health Policy and Planning 23(4):252-263

Ensor T (2004) Informal payments for health care in transition economies. Soc Sci Med 58(2):237-246

Fu R, Wang Y, Bao H, Wang Z, Li Y et al (2014) Trend of urban-rural disparities in hospital admissions and medical expenditure in China from 2003 to 2011. PLoS One 9(9):e108571. doi:10.1371/journal.pone.0108571

Ha NTH, Berman P, Larsen U (2002) Household utilization and expenditure on private and publichealth services in Vietnam. Health Policy Plan 17(1):61-70

Hort K (2011) Private hospitals in Vietnam recent growth and role in the health sector. Policy Brief Series, Nossal Institute for Global Health, June. http://ni.unimelb.edu.au/_data/assets/pdf_file/0004/526927/private-hospitals-in-vietnam. pdf

Hu S, Tang S, Liu Y, Zhao Y, Escobar ML et al (2008) Reform of how health care is paid for in China: challenges and opportunities. Lancet 372:1846-1853

Jelicic Kadic A, Zanic M, Skaricic N, Marusic A (2014) Using the WHO essential medicines list to assess the appropriateness of insurance coverage decisions: a case study of the Croatian national medicine reimbursement list. PLoS One 9(10):e111474. doi:10.1371/journal.pone.0111474

Karan A, Selvaraj S, Mahal A (2014) Moving to universal coverage? Trends in the burden of out-of-pocket payments for health care across social groups in India, 1999-2000 to 2011-12. PLoS One 9(8):e105162. doi:10.1371/journal. pone.0105162

Kim JH, Lee KS, Yoo KB, Park EC (2015) The differences in health care utilization between medical aid and health insurance: a longitudinal study using propensity score matching. PLoS One 10(3):e0119939. doi:10.1371/journal. pone.0119939

Leonard KL, Mliga GR, Mariam DH (2002) Bypassing health centres in Tanzania: revealed preferences for quality. J Afr Econ 11(4):441-471

Liu X, Tang S, Yu B, Phuong NK, Yan F, Thien DD, Tolhurst R (2012) Can rural health insurance improve equity in health care utilization? A comparison between China and Vietnam. Int J Equity Health 11:10

Loganathan T, Lee W-S, Lee K-F, Jit M, Ng C-W (2015) Household catastrophic healthcare expenditure and impoverishment due to rotavirus gastroenteritis requiring hospitalization in Malaysia. PLoS One 10(5):e0125878. doi:10.1371/ journal.pone.0125878

Lönnroth K, Tran TU, Thuong LM, Quy HT, Diwan V (2001) Can I afford free treatment?: perceived consequences of health care provider choices among people with tuberculosis in Ho Chi Minh City, Vietnam. Soc Sci Med 52(6):935-948

Mao W, Vu H, Xie Z, Chen W, Tang S (2015) Systematic review on irrational use of medicines in China and Vietnam. PLoS One 10(3):e0117710. doi:10.1371/journal.pone.0117710

Ngoc Q (2015) Benh nhan bo bao hiem y te, vuot tuyen. Tien Phong, 22 January. Retrieved 18 June 2015. http://www. tienphong.vn/xa-hoi/benh-nhan-bo-bao-hiem-y-te-vuot-tuyen-813484.tpo

Phuong N (2015) Bo Y Te: 70\% so benh vuot tuyen gay lang phi. VnExpress, 9 January. Retrieved 18 June 2015. http:// vnexpress.net/tin-tuc/thoi-su/bo-y-te-70-so-benh-vuot-tuyen-gay-lang-phi-3131097.html

Nguyen KT, Khuat OTH, Ma S, Pham DC, Khuat GTH, Ruger JP, (2012) Coping with health care expenses among poor households: evidence from a rural commune in Vietnam. Soc Sci Med 74(5):724-733

Parkhurst JO, Ssengooba F (2009) Assessing access barriers to maternal health care: measuring bypassing to identify health centre needs in rural Uganda. Health Policy Plan 24:377-384

Paul BK (1999) National health care 'by-passing' in Bangladesh: a comparative study. J Rural Health 49(5):679-689 
Russell S (2004) The economic burden of illness for households in developing countries: a review of studies focusing on malaria, tuberculosis, and human immunodeficiency virus/acquired immunodeficiency syndrome. Am J Trop Med Hyg 71(2):147-155

Santatiwongchai B, Chantarastapornchit V, Wilkinson T, Thiboonboon K, Rattanavipapong W, Walker DG et al (2015) Methodological variation in economic evaluations conducted in low- and middle-income countries: information for reference case development. PLoS One 10(5):e0123853. doi:10.1371/journal.pone.0123853

Segall M, Tipping G, Lucas H, Dung TV, Tam NT, Vinh DX, Huong DL (2002) Economic transition should come with a health warning: the case of Vietnam. J Epidemiol Community Health 56(7):497-505

Selvaraj SNV (2010) Access to medicine in India: issues challenges and policy options. In: Mahal A, Debroy B, Bhandari L (eds) India: health report 2010. Indicus Analytics, Business Standard 2010, New Delhi

Sepehri A, Chernomas R, Akram-Lodhi AH (2003) If they get sick, they are in trouble: health care restructuring, user charges, and equity in Vietnam. Int J Health Serv 33(1):137-161

Sepehri A, Chernomas R, Akram-Lodhi H (2005) Penalizing patients and rewarding providers: user charges and health care utilization in Vietnam. Health Policy Plan 20(2):90-99

Thuan NT, Lofgren C, Lindholm L, Chuc NT (2008) Choice of healthcare provider following reform in Vietnam. BMC Health Servi Res 8:162

Tran BX, Nong VM, Maher RM, Nguyen PK, Luu HN (2014) A systematic review of scope and quality of health economic evaluation studies in Vietnam. PLoS One 9(8):e103825. doi:10.1371/journal.pone.0103825

Van Doorslaer E, O'Donnell O, Rannan-Eliya RP, Somanathan A, Adhikari SR, Garg CC, Zhao Y (2006) Effect of payments for health care on poverty estimates in 11 countries in Asia: an analysis of household survey data. Lancet 368(9544):1357-1364

Van Doorslaer E, O'Donnell O, Rannan-Eliya RP, Somanathan A, Adhikari SR, Garg CC, Zhao Y et al. (2007) Catastrophic payments for health care in Asia. Health Econ 16(11):1159-1184

Whitehead M, Dahlgren G, Evans T (2001) Equity and health sector reforms: can low-income countries escape the medical poverty trap? Lancet 358(9284):833-836

Xu K, Evans DB, Carrin G, Aguilar-Rivera AM, Musgrove P, Evans T (2007) Protecting households from catastrophic health spending. Health Aff 26(4):972-983

Zhang Y, Tang W, Zhang X, Zhang Y, Zhang L (2015) National Health Insurance Development in China from 2004 to 2011 : coverage versus benefits. PLoS One 10(5):e0124995. doi:10.1371/journal.pone.0124995

\section{Submit your manuscript to a SpringerOpen ${ }^{\circ}$ journal and benefit from:}

- Convenient online submission

- Rigorous peer review

- Immediate publication on acceptance

- Open access: articles freely available online

- High visibility within the field

- Retaining the copyright to your article

Submit your next manuscript at $\boldsymbol{s p r i n g e r o p e n . c o m ~}$ 